\title{
Lumi-Map, a Real-Time Luciferase Bioluminescence Screen of Mutants Combined with MutMap, Reveals Arabidopsis Genes Involved in PAMP-Triggered Immunity
}

\author{
Hiroaki Kato, ${ }^{1,2}$ Kiyoshi Onai, ${ }^{2}$ Akira Abe, ${ }^{1}$ Motoki Shimizu, ${ }^{1}$ Hiroki Takagi, ${ }^{1}$ Chika Tateda, ${ }^{1}$ \\ Hiroe Utsushi, ${ }^{1}$ Suthitar Singkarabanit-Ogawa, ${ }^{3}$ Saeko Kitakura, ${ }^{3}$ Erika Ono, ${ }^{3}$ Cyril Zipfel,,${ }^{4,5}$ \\ Yoshitaka Takano, ${ }^{3}$ Masahiro Ishiura, ${ }^{6}$ and Ryohei Terauchi ${ }^{1,2,+}$ \\ ${ }^{1}$ Iwate Biotechnology Research Center, Kitakami, Japan \\ ${ }^{2}$ Laboratory of Crop Evolution, Graduate School of Agriculture, Kyoto University, Kyoto, Japan \\ ${ }^{3}$ Laboratory of Plant Pathology, Graduate School of Agriculture, Kyoto University, Kyoto, Japan \\ ${ }^{4}$ The Sainsbury Laboratory, University of East Anglia, Norwich Research Park, Norwich, U.K. \\ ${ }^{5}$ Institute of Plant and Microbial Biology, Zurich-Basel Plant Science Center, University of Zurich, Zurich, Switzerland \\ ${ }^{6}$ Center for Gene Research, Nagoya University, Nagoya, Japan
}

Accepted 1 September 2020.

Plants recognize pathogen-associated molecular patterns (PAMPs) to activate PAMP-triggered immunity (PTI). However, our knowledge of PTI signaling remains limited. In this report, we introduce Lumi-Map, a high-throughput platform for identifying causative single-nucleotide polymorphisms (SNPs) for studying PTI signaling components. In Lumi-Map, a transgenic reporter plant line is produced that contains a firefly luciferase $(L U C)$ gene driven by a defense gene promoter, which generates luminescence upon PAMP treatment. The line is mutagenized and the mutants with altered luminescence patterns are screened by a high-throughput real-time bioluminescence monitoring system. Selected mutants are subjected to MutMap analysis, a whole-genome sequencingbased method of rapid mutation identification, to identify the causative SNP responsible for the luminescence pattern change. We generated nine transgenic Arabidopsis reporter

Accession numbers: The accession numbers for Arabidopsis genes discussed in this article are ERF019 (AT1G22810), HSI2/VAL1 (AT2G30470), WRKY29 (AT4G23550), THO5 (AT5G42920), FLS2 (AT5G46330), and CDK8 (AT5G63610).

Current address of H. Takagi: Graduate School of Bioresources and Environmental Science, Ishikawa Prefectural University, Nonoichi, Japan.

${ }^{\dagger}$ Corresponding author: R. Terauchi; terauchi@ibrc.or.jp

Funding: Support was provided by the Gatsby Charitable Foundation to C. Zipfel, Universität Zürich to C. Zipfel, Japan Society for the Promotion of Science grant $15 \mathrm{H} 05779$ to R. Terauchi and grant $18 \mathrm{H} 02204$ to Y. Takano, Schweizerischer Nationalfonds zur Förderung der Wissenschaftlichen Forschung grant 31003A_182625 to C. Zipfel, H2020 European Research Council grant 773153 to C. Zipfel, and Asahi Glass Foundation to Y. Takano.

*The $\boldsymbol{e}$-Xtra logo stands for "electronic extra" and indicates that supplementary tables, supplementary figures, and supplementary materials are published online.

The author(s) declare no conflict of interest.

(c) (1) $\circledast \Theta$ Copyright $(02020$ The Author(s). This is an open access article distributed under the CC BY-NC-ND 4.0 International license. lines expressing the $L U C$ gene fused to multiple promoter sequences of defense-related genes. These lines generate luminescence upon activation of FLAGELLIN-SENSING 2 (FLS2) by flg22, a PAMP derived from bacterial flagellin. We selected the WRKY29-promoter reporter line to identify mutants in the signaling pathway downstream of $F L S 2$. After screening 24,000 ethylmethanesulfonate-induced mutants of the reporter line, we isolated 22 mutants with altered WRKY29 expression upon flg22 treatment (abbreviated as awf mutants). Although five flg22-insensitive awf mutants harbored mutations in FLS2 itself, Lumi-Map revealed three genes not previously associated with PTI. Lumi-Map has the potential to identify novel PAMPs and their receptors as well as signaling components downstream of the receptors.

Keywords: Arabidopsis thaliana, elicitors, genetics and gene regulation, high-throughput phenotyping, luciferase reporter, MAMPs, MutMap, PAMP-triggered immunity, plant defense mechanisms, receptor

To defend themselves against pathogens, plants must recognize them and activate appropriate immune responses. Initial recognition of pathogens is mediated by pattern recognition receptors (PRRs) localized at the plasma membrane that sense pathogen-associated molecular patterns (PAMPs) (Boutrot and Zipfel 2017). PAMP-triggered immunity (PTI) confers broadrange resistance against pathogens (Boutrot and Zipfel 2017). Flg22, a 22-amino-acid peptide derived from bacterial flagellin, is one of the most extensively studied PAMPs in plants (Boller and Felix 2009). Flg22 is recognized by the PRR FLAGELLINSENSING 2 (FLS2), a leucine-rich repeat receptor kinase (Gómez-Gómez and Boller 2000). FLS2 associates with the coreceptor BRASSINOSTEROID INSENSITIVE 1-ASSOCIATED RECEPTOR KINASE 1 (BAK1) and related SOMATIC EMBRYOGENESIS RECEPTOR KINASES in an flg22dependent manner (Chinchilla et al. 2007; Heese et al. 2007; Schulze et al. 2010; Sun et al. 2013). Among other receptor-like cytoplasmic kinases, BOTRYTIS-INDUCED KINASE 1 associates with FLS2 (Lu et al. 2010; Zhang et al. 2010), and 
activates the NADPH oxidase RESPIRATORY BURST OXIDASE HOMOLOG PROTEIN D (RBOHD) upon PAMP binding, which leads to the apoplastic production of reactive oxygen species (ROS) in Arabidopsis (Kadota et al. 2014; L. Li et al. 2014). PAMP-mediated ROS burst is also regulated by $\mathrm{Ca}^{2+}$-dependent protein kinase-mediated phosphorylation (Boudsocq et al. 2010; Dubiella et al. 2013). Mitogen-activated protein kinase (MAPK) cascades involving MPK3 and MPK6 are also activated and control PTI signaling at multiple levels (Rasmussen et al. 2012). Upon PAMP perception, several transcription factors involved in defense gene regulation, including those belonging to the WRKY and ethylene responsive factor (ERF) families, are phosphorylated and activated by MAPKs (Birkenbihl et al. 2017; Rasmussen et al. 2012). However, a complete molecular picture of the signaling networks regulating the rapid reprogramming of immunity-related genes remains elusive.

Forward genetic approaches have been extensively used to identify PTI signaling components. The elf18-insensitive mutants, impaired in ROS production after elf18 treatment, included mutants of proteins involved in endoplasmic reticulum (ER) quality control (J. Li et al. 2009; Nekrasov et al. 2009). Boutrot et al. (2010) isolated 21 flagellin-insensitive (fin) mutants showing alterations in flg22-induced ROS burst, identifying mutants including finl (corresponding to FLS2), fin2 (BAK1), fin3 (a mutant of EIN2 which encodes a central regulator of ethylene-mediated signaling) (Boutrot et al. 2010), and fin4 (a mutant in the gene encoding aspartate oxidase) (Macho et al. 2012). Ranf et al. (2012) screened mutant lines of Arabidopsis with an aequorin reporter transgene which showed changes in calcium elevation after flg22 treatment, resulting in the isolation of 35 changed calcium elevation (cce) mutants (Ranf et al. 2012). In Arabidopsis seedlings, sucrose-induced flavonoid accumulation is repressed upon exposure to different PAMPs (Saijo et al. 2009; Serrano et al. 2012). Using this readout, more than 50 priority in sweet life ( $p s l)$ mutants were isolated, including mutants in several genes involved in ER quality control (Lu et al. 2009; Saijo et al. 2009) as well as psl6, an allele of ein2 (Tintor et al. 2013). Monaghan et al. (2014) performed a screen of mutants in the Arabidopsis bakl-5 background (modifier of bak1-5 [mob]) to identify mutants that restored PAMP-triggered ROS production. This screen identified the allelic mob1 and mob2 mutants in CPK28 (Monaghan et al. 2014) as well as mob6, a mutant of the gene encoding SITE-1 PROTEASE that controls the cleavage of the endogenous peptide RAPID ALKALINIZATION FACTOR 23 to regulate PTI signaling (Stegmann et al. 2017). Furthermore, F. Li et al. (2014) reported a screening system named "Arabidopsis genes governing immune gene expression" (aggie), in which the promoter of FLG22-INDUCED RECEPTOR-LIKE KINASE 1 (FRKl) was fused with the firefly luciferase (LUC) gene and its induction after flg22 treatment was monitored with a luminometer. This system identified two mutants: aggiel, a mutant of RNA POLYMERASE II C-TERMINAL DOMAIN PHOSPHATASE-LIKE 3 (F. Li et al. 2014), and aggie2, a mutant of POLY(ADP-RIBOSE) GLYCOHYDROLASE 1, revealing that protein poly ADP-ribosylation plays a role in defense gene expression (Feng et al. 2015). These previous forward genetics studies of PTI signaling all involved conventional map-based cloning or a combination of map-based cloning and whole-genome sequencing (WGS).

Real-time bioluminescence monitoring of an organism with a reporter transgene driven by a promoter of interest is a powerful tool suitable for large-scale analyses. In this approach, gene expression is monitored in real time with high sensitivity and accuracy in a nondestructive manner. By taking advantage of this platform, 35 Arabidopsis circadian rhythm mutants were isolated after screening 100,000 seedlings for expression of an $L U C$ gene fused to the promoters of GIGANTEA and FLOWERING LOCUS T, which show circadian expression. This study resulted in the successful isolation of PHYTOCLOCK1, an essential component of the Arabidopsis circadian clock (Onai and Ishiura 2005; Onai et al. 2004).

Recent developments in next-generation sequencing (NGS) technologies is accelerating the WGS-based identification of mutations underlying interesting phenotypes. MutMap is one such WGS-based technique (Abe et al. 2012). In MutMap, a mutant is crossed to the original wild-type line and propagated until $F_{2}$ progeny are obtained. DNA from 20 to $30 F_{2}$ progeny displaying the mutant phenotype are pooled and subjected to WGS. The resulting NGS short reads are aligned to the reference genome sequence of the wild-type line. A genomic region aligned to short reads with a higher frequency of singlenucleotide polymorphisms (SNPs) points to the position of the causative mutation responsible for the phenotype. MutMap can identify the causative SNP in a mutant in a single WGS run, allowing for rapid and low-cost mutation identification.

In this report, we demonstrate Lumi-Map, which combines automated real-time luciferase bioluminescence monitoring for the high-throughput screening of mutants with MutMap to identify causative mutations involved in PTI signaling (Fig. 1). We generated nine Arabidopsis reporter lines that respond to the flg22 treatment. A screen of 24,000 Arabidopsis mutant lines with a transgenic $L U C$ gene driven by the WRKY29 gene promoter resulted in the isolation of 22 mutants with altered WRKY29 expression upon flg22 treatment (awf mutants). The subsequent application of MutMap enabled the identification of mutated genes responsible for five mutants potentially involved in PTI.

\section{RESULTS}

\section{Generation of transgenic Arabidopsis reporter lines.}

We generated Arabidopsis $L U C$ reporter lines with promoters from nine genes (Supplementary Table S1) potentially involved in defense responses (Asai et al. 2002; Denoux et al. 2008; He et al. 2006; Navarro et al. 2004; Zipfel et al. 2004, 2006). Realtime bioluminescence monitoring revealed that these lines under liquid culture exhibited different luminescence patterns after treatment with flg22 (Fig. 2). Four lines (WRKY29, WRKY18, WRKY28, and RBOHD) showed a transient induction of luminescence followed by suppression, whereas three (PAL1, At1g51890, and At2g17740) showed an induction followed by a lasting expression, and the rest (PR4 and ERF1) showed gradual increase in luminescence over the time.

\section{Screening Arabidopsis mutants affected in flg22-induced WRKY29 expression.}

From among the nine reporter lines, we focused on the WRKY29 reporter line to establish Lumi-Map because previous studies indicate its role in defense response; expression of WRKY29 is induced during flg22-triggered PTI (flg22-PTI) (Asai et al. 2002; Eulgem et al. 2000) and overexpression of WRKY29 in Arabidopsis enhances resistance to Pseudomonas syringae pv. maculicola (Asai et al. 2002). WRKY29 transcription is also known to be regulated by defense-related MAPKs such as MPK3 and MPK6 in Arabidopsis (Asai et al. 2002). In addition, WRKY 29 reporter showed a quick and transient response against flg22 treatment and also exhibited a clear signal-to-noise ratio (Supplementary Fig. S1). We used the WRKY29 promoter sequence $(-1,931$ to -1$)$ (Serrano et al. 2007) to establish a high-throughput method for monitoring gene expression during flg22-PTI. The homozygous Arabidopsis reporter line carrying the $\mathrm{P}_{W R K Y 29}:: L U C$ transgene was 
named W29-1-4 (Supplementary Fig. S1A), which showed a transient induction of luciferase-mediated bioluminescence under liquid culture after flg22 treatment (Supplementary Figs. S1B and S2). A similar result was obtained from the independent transgenic line W29-114A, which carried the same

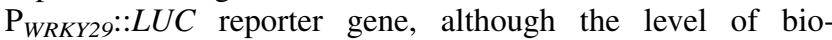
luminescence differed (Supplementary Fig. S1B). We also examined the level of WRKY29 mRNA during PTI by quantitative reverse-transcription PCR (qRT-PCR) after flg22 treatment. This qRT-PCR result was overall consistent with the bioluminescence data from the W29-1-4 reporter line after treatment with flg22 (Supplementary Fig. S1C), indicating that it is possible to monitor WRKY29 transcription levels by the bioluminescence.

To identify mutants showing altered response to flg22, we prepared ethylmethanesulfonate (EMS)-mutagenized seed of the W29-1-4 reporter line and screened 24,000 $\mathrm{M}_{2}$ seedlings with a high-throughput, real-time, bioluminescence monitoring system following flg22 treatment (Fig. 1). In total, we isolated 263 candidate awf mutants and confirmed their bioluminescence phenotypes in $\mathrm{M}_{3}$ progeny. We selected plants showing less than $50 \%$ or more than $200 \%$ of the maximum bioluminescence level of the original reporter line as candidate mutants. For a subset of mutant lines, $\mathbf{M}_{3}$ seed could not be obtained due to abnormal growth or infertility. Mutant lines with significantly smaller seedlings than the parental line were excluded from further analysis. Finally, 22 awf mutants, including 18 with lower and 4 with higher bioluminescence than the wild type, were isolated (Fig. 3; Supplementary Fig. S3; Table 1). Among the high-luminescence awf mutants, awf 21 showed a slightly delayed induction (Fig. 3D).

\section{Classification of the $a w f$ mutants.}

The isolated awf mutant lines may have mutations in the signaling pathway specific to flg22 perception by FLS2 or in a pathway shared by several PAMPs. To address this question, we

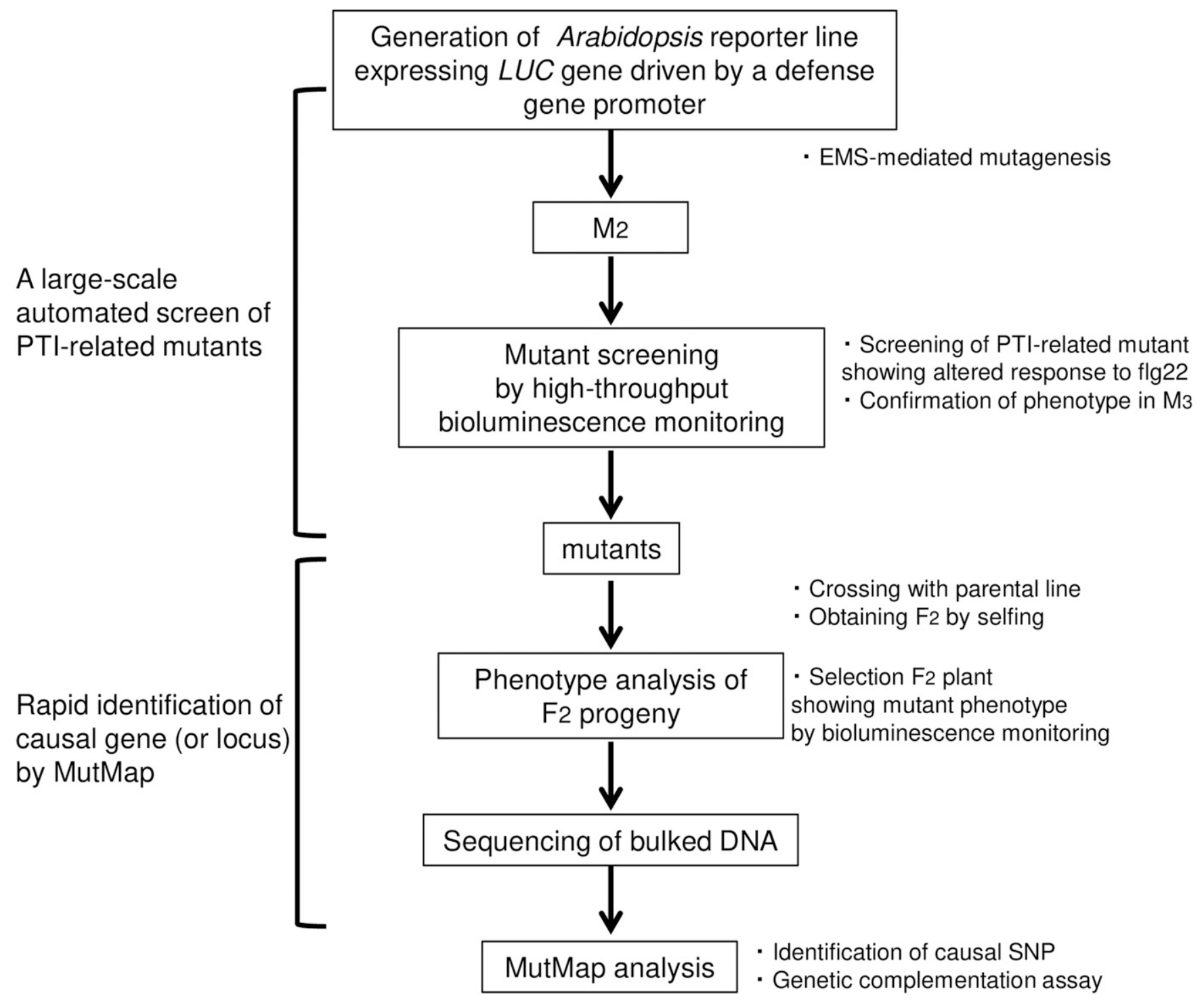

Fig. 1. Diagram of the Lumi-Map method. A WRKY29 reporter strain (W29-1-4) was mutagenized with ethylmethanesulfonate (EMS) and M 2 progeny were obtained. Mutant screening was performed by bioluminescence monitoring of flg22-treated $\mathrm{M}_{2}$ seedlings. PTI = pathogen-associated molecular patterntriggered immunity. $\mathbf{M}_{2}$ seedlings that showed a mutant bioluminescence phenotype were propagated to $\mathbf{M}_{3}$, and mutants with altered WRKY29 expression upon flg22 treatment ( $a w f$ mutants) were then chosen after confirmation of the bioluminescence phenotypes. Identification of the causal gene was performed by MutMap. The awf mutants were crossed to the parental reporter line (W29-1-4) and $\mathrm{F}_{1}$ progeny were selfed to obtain the $\mathrm{F}_{2} . \mathrm{F}_{2}$ plants were treated with flg22 and their bioluminescence phenotypes were monitored. DNA of $F_{2}$ plants showing mutant phenotypes were subjected to whole-genome sequencing followed by MutMap analysis to identify causal single-nucleotide polymorphisms (SNPs). 
studied the responses of awf mutants to two other PAMPs: elf18, derived from bacterial elongation factor Tu (EF-Tu), and chitin, a component of fungal cell walls. For both PAMPs, the cognate PRRs have been isolated: EF-TU RECEPTOR for elf18 and LYSIN MOTIF RECEPTOR KINASE 5 acting together with CHITIN ELICITOR RECEPTOR KINASE 1 for chitin (Cao et al. 2014; Miya et al. 2007; Wan et al. 2008; Zipfel et al. 2006). Similar to its response to flg22 treatment, the W291-4 reporter line showed a transient induction of bioluminescence following elf18 and chitin treatment (Supplementary Fig. S2). Therefore, we treated the $a w f$ mutants with elf18 and chitin and grouped them based on their response to the three different PAMPs.
The low-bioluminescence mutants were classified into four groups depending on their responses to flg22, elf18, and chitin (Fig. 4A). Five mutants (awf3, awf4, awf7, awf9, and awf18) showed no induction of bioluminescence after flg22 treatment, while induction after elf18 and chitin treatment was not significantly altered (group I). Three mutants (awf11, awf12, and awf17) showed low bioluminescence induction after flg22 treatment, while the induction by elf 18 and chitin was unaltered (group II). Three mutants ( $a w f 6, a w f 13$, and $a w f 16$ ) showed low bioluminescence induction after both flg 22 and elf 18 treatment but normal induction by chitin (group III). The remaining seven mutants (awf1, awf2, awf5, awf8, awf10, awf14, and awf15) showed low bioluminescence induction against all three
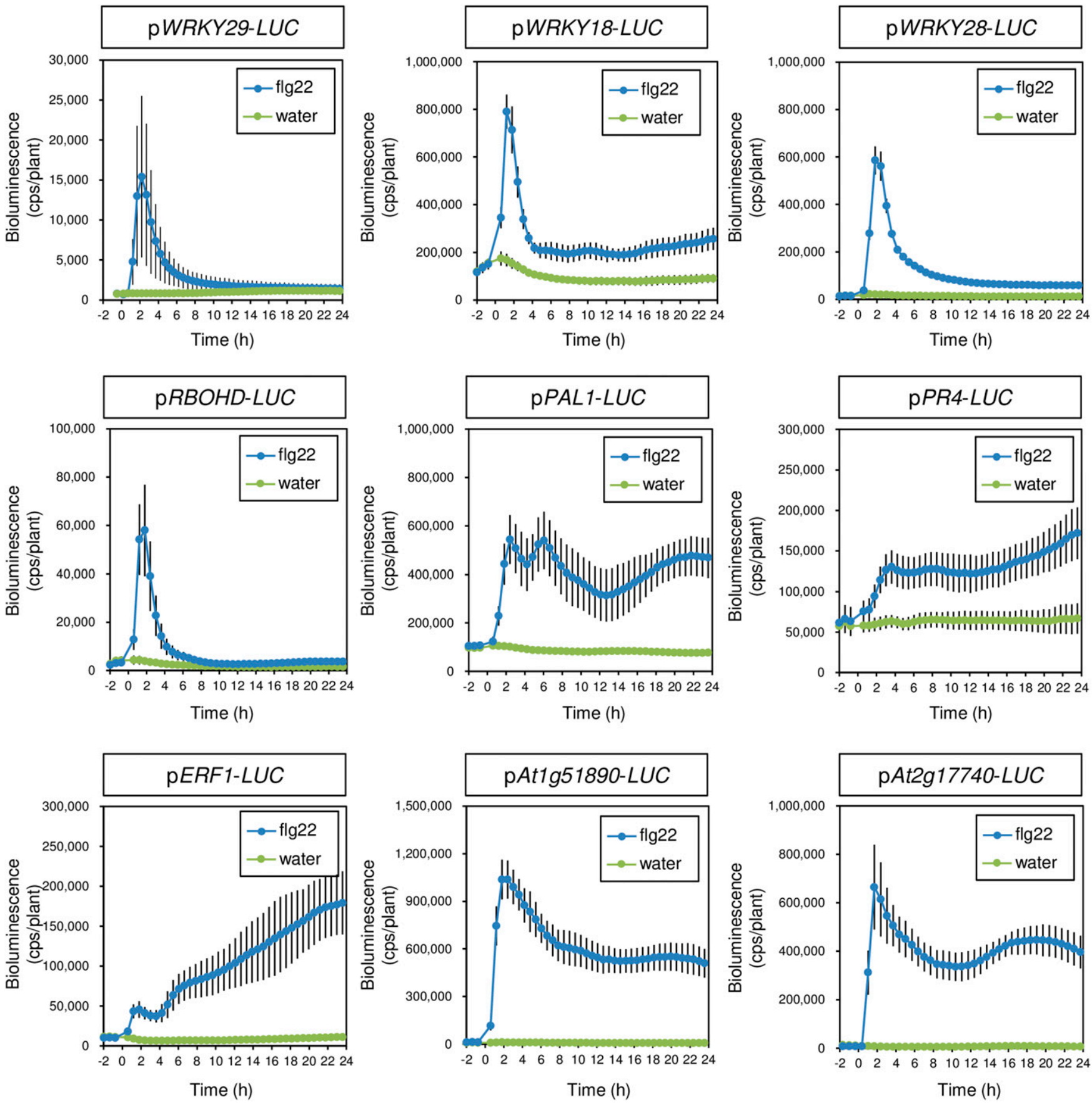

Fig. 2. Bioluminescence patterns of nine Arabidopsis reporter lines after treatment with flg22. Eight-day-old seedlings of transgenic Arabidopsis plants

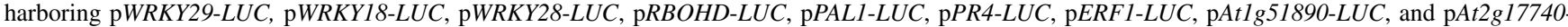
$L U C$ were treated with water (green) or $0.5 \mu \mathrm{M}$ flg22 (blue). Bioluminescence from each seedling was monitored with a real-time bioluminescence monitoring system at the indicated time points. Data are shown as mean \pm standard error from at least seven seedlings per treatment. 
PAMPs tested (group IV). All four mutants with a higher bioluminescence level after flg22 treatment (awf19, awf20, awf21, and $a w f 22$ ) showed higher bioluminescence levels after elf18 and chitin treatments as well (Fig. 4B).

\section{Isolation of $F L S 2$ mutants.}

FLS2 is the PRR for flg22 (Gómez-Gómez and Boller 2000). In the $f l s 2$ mutant, PTI responses such as ROS production and MAPK phosphorylation do not occur following flg22 treatment (Asai et al. 2002; Felix et al. 1999). Five awf mutants showed no response after flg22 treatment (group I) (Fig. 4A), which led us to ask whether these mutants had mutations in the FLS2 gene itself. The coding region of $F L S 2$ was amplified from genomic DNA from the group I mutants (awf3, awf4, awf7, awf9, and awf18) by PCR and sequenced. Nucleotide substitutions were detected in the FLS2 gene in all five group I mutants (Fig. 5). Although they were independently isolated from different $\mathbf{M}_{2}$ pools, $a w f 3, a w f 7$, and $a w f 9$ all shared the same mutation in the kinase domain of FLS2 (G1042E). Further DNA sequencing of selected regions of genomes of the mutants revealed that awf3 and $a w f 7$ are identical, presumably caused by contamination of seed, whereas awf9 is different from them (Supplementary Table S2). Complementation experiments confirmed that the flg22-insensitive phenotype of awf4, awf9, and awf18 were caused by loss of FLS2 function (Supplementary Fig. S4). Thus, we conclude that mutants showing no bioluminescence activation after flg22 treatment but with unaltered responses to other PAMPs are $f l s 2$ mutant alleles. The identification of $F L S 2$ mutants in our genetic screen suggests that the real-time bioluminescence monitoring platform allows uncovering PTIrelated genes, including those encoding PRRs.

\section{MutMap identifies causative genomic regions of $a w f$ mutants.}

We first applied MutMap (Abe et al. 2012) to three awf mutants (awf1, awf2, and awf9) that showed no or low bioluminescence induction after flg 22 treatment. The awf9 mutant containing a mutation in $f l s 2$ (Fig. 5) was included as a positive control. We crossed each mutant to the W29-1-4 reporter line and obtained $F_{1}$ seed. The resulting $F_{1}$ plants were self-pollinated

\section{A awf1}

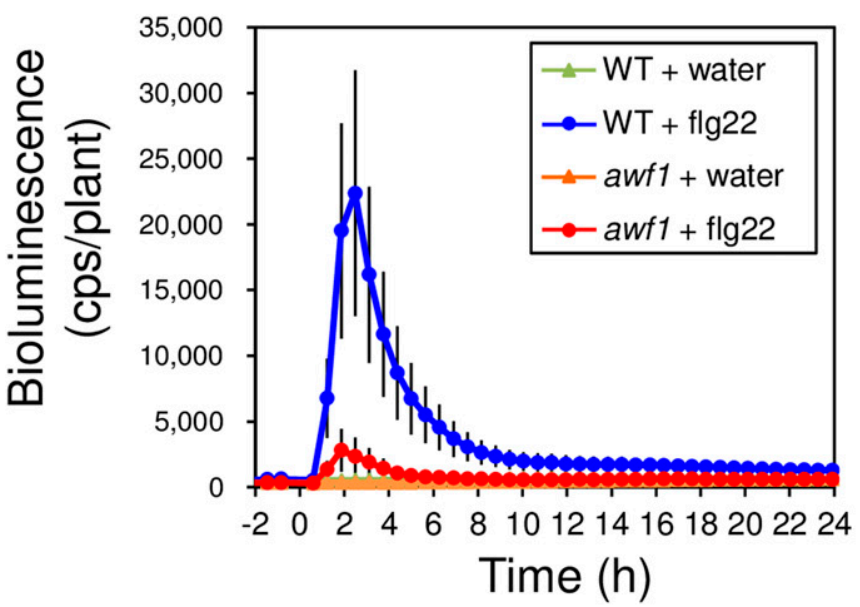

\section{C awf9}

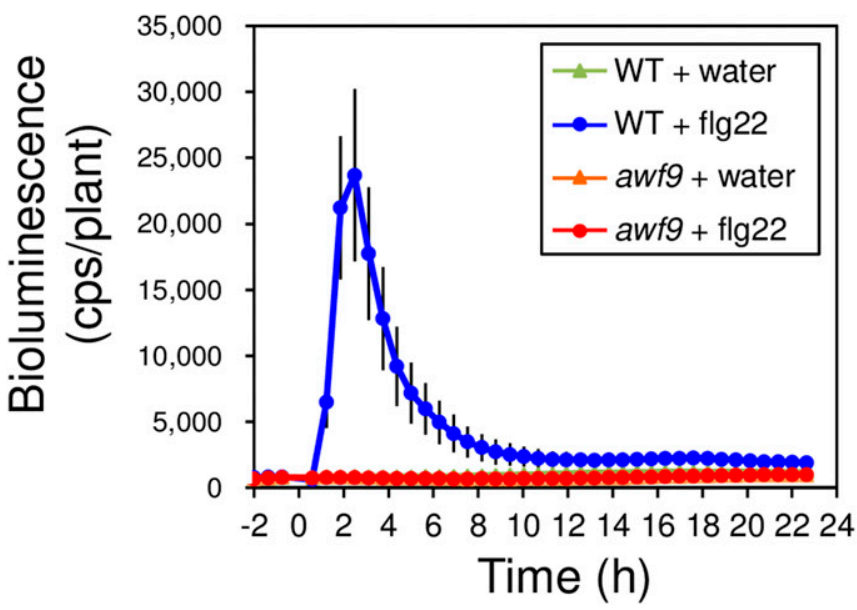

\section{B awf2}

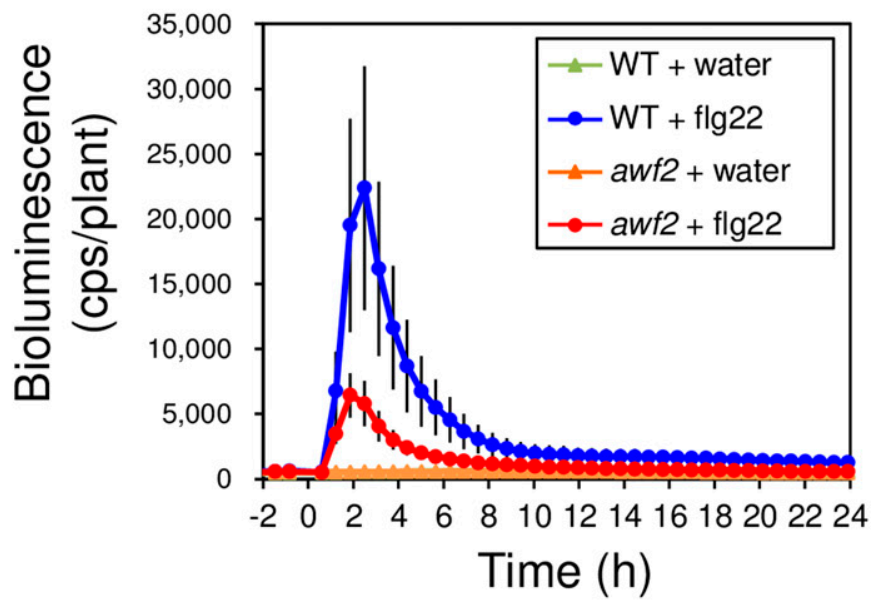

\section{D awf21}

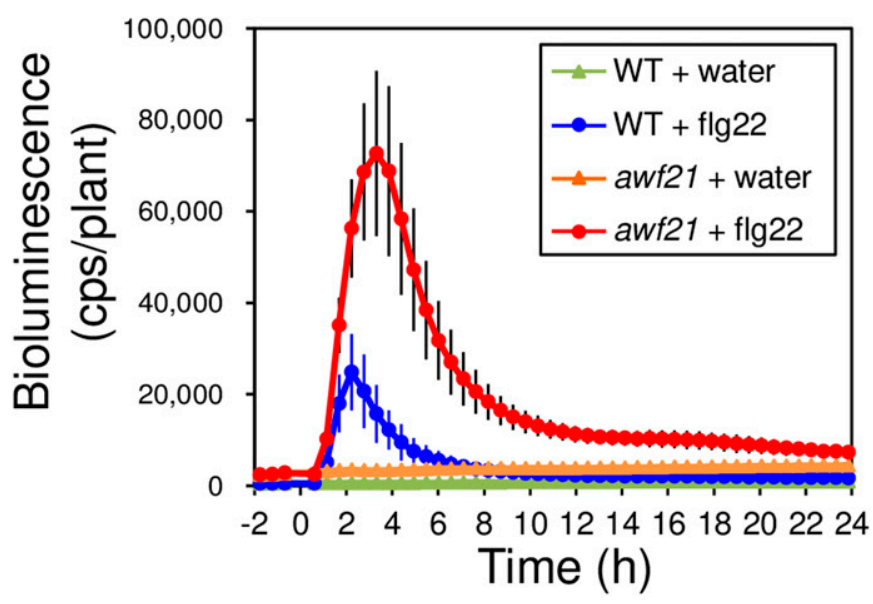

Fig. 3. Bioluminescence patterns of mutants with altered responses to flg 22 treatment. Eight-day-old seedlings of the wild type (W29-1-4) (WT) and mutants treated with water or $0.5 \mu \mathrm{M}$ flg22. Bioluminescence from each seedling was monitored with a real-time bioluminescence monitoring system at the indicated time points. Data are shown as mean \pm standard error from at least 14 seedlings per treatment. Experiments were conducted three times with similar results. A, A low-bioluminescence mutant with altered WRKY29 expression upon flg22 treatment (awf mutant), awf1. B, A low-bioluminescence mutant, $a w f 2$. C, A noresponse mutant, awf9. D, A high-bioluminescence and peak time-altered mutant, awf 21 . 
and their $F_{2}$ progeny were treated with flg22 and monitored for bioluminescence activation. We observed segregation of the wild-type and mutant flg22-induced bioluminescence phenotypes in an approximate 3:1 ratio (Supplementary Fig. S5; Supplementary Table S3), suggesting that all three mutants were caused by single-locus, recessive mutations. Following the MutMap procedure, we pooled DNA from $30 \mathrm{~F}_{2}$ individuals displaying the mutant phenotype. For MutMap analysis of lowbioluminescence awf mutants, we used $30 \quad \mathrm{~F}_{2}$ individuals showing the lowest bioluminescence at the peak time after flg22 treatment (Supplementary Fig. S5). Equal amounts of leaf material were obtained from each individual and mixed together, and DNA was extracted from this mixture. This pooled DNA was subjected to WGS using an Illumina NGS platform. We obtained 25 to 30 million sequence reads from each of the three mutants (Supplementary Table S4). These reads were aligned to the W29-1-4 reference sequence (average read depth: 25.1, range of read depths: 13.8-45.1) (Supplementary Table S4) and SNPs were identified. For each genomic position containing an SNP, the frequency of short reads containing SNPs different from the reference (SNP index) was calculated and graphs relating SNP positions to SNP indices were generated for the five Arabidopsis chromosomes (Fig. 6; Supplementary Table S5). For all three mutants, we identified single genomic regions harboring a cluster of SNPs with an SNP index $>0.95$; awf1 showed an SNP-index peak on chromosome 1, whereas $a w f 2$ and awf9 had SNP-index peaks on chromosome 5 (Fig. 6). The position of the SNP-index peak of awf9 exactly corresponded to the location of FLS2 (SNP-18794926 in chromosome 5). These results demonstrate that Lumi-Map (bioluminescence monitoring combined with MutMap) rapidly and effectively identifies the position of causative mutations for mutants with altered responses to flg22 treatment.

Among the other 11 low-bioluminescence mutants that seem to be non- $f l s 2$ mutants, we extended MutMap to four other mutants (awf5, awf8, awf14, and awf16). We observed segregation in the $\mathrm{F}_{2}$ individuals (Supplementary Fig. S5; Supplementary Table S3). We then pooled DNA from $30 \mathrm{~F}_{2}$ mutant progeny and subjected this DNA to MutMap. In all four cases, we identified single genomic regions with SNP-index peaks: awf5 and awf14 showed SNP-index peaks on chromosome 5, whereas awf8 and awf16 had SNP-index peaks on chromosome 2 (Supplementary Fig. S6). Of the seven low-bioluminescence mutants applied to MutMap, none of the loci identified by MutMap overlapped (Fig. 6; Supplementary Fig. S6), suggesting that different mutations are responsible for the observed phenotypes.

We also performed MutMap on awf19 and awf21, which showed higher bioluminescence upon flg22 treatment and observed segregation in the $\mathrm{F}_{2}$ between plants with wild-type and higher bioluminescence levels (Supplementary Fig. S5; Supplementary Table S3). For MutMap analysis of high-bioluminescence awf mutants, we used $30 \mathrm{~F}_{2}$ individuals showing the highest bioluminescence at the peak time after flg22 treatment (Supplementary Fig. S5). MutMap identified a single genomic region harboring an SNP-index peak on chromosome 5 for awf19 and chromosome 1 for awf 21 (Supplementary Fig. S6).

\section{Identification of the causative mutations of selected $a w f$ mutants.}

To identify the candidate causative SNPs in the genomic region with a peak of SNP-index plot, we selected mutations fitting to the following criteria: (i) mutations with stronger effects (mutation causing stop codon $>$ mutation causing amino acid substitution $>$ mutation in the promoter region or untranslated region), (ii) mutations proximal to the peak of the SNP-index plot, and (iii) mutations with SNP-index values close to 1. For the awfl mutant (group IV), we examined SNPs with an SNP index > 0.95 in detail (Supplementary Table S6). One SNP, SNP-8074904, was a nonsense mutation located in ERF019 (AT1G22810) changing a Trp residue to a stop codon (Fig. 7A and B; Supplementary Table S6). The ERF family in Arabidopsis consists of 12 groups and ERFO19 is a member of group IIc (Nakano et al. 2006). A class IIc ERF in rice, SERF1, is reported to be involved in ROS signaling during salt stress response (Schmidt et al. 2013). Therefore, we hypothesized that the nonsense mutation in ERF019 underlies the awf1 mutant. To test this hypothesis, we carried out a complementation assay by transforming the awf1 mutant with wild-type ERF019 under the control of its native promoter. Bioluminescence following

Table 1. Mutants with altered WRKY29 expression upon flg22 treatment (awf mutants) and their bioluminescence phenotypes

\begin{tabular}{|c|c|c|c|c|c|}
\hline Mutant & Reporter & Generation & Type $^{\mathbf{a}}$ & Bioluminescence $(\%)^{\mathbf{b}}$ & $n$ \\
\hline awfl & $\mathrm{P}_{W R K Y 29}:: L U C$ & M5 & Low & $12.5 \pm 7.3$ & 24 \\
\hline awf2 & $\mathrm{P}_{\text {WRKY29: }: L U C}$ & M5 & Low & $28.8 \pm 7.6$ & 23 \\
\hline awf 3 & $\mathrm{P}_{W R K Y 29}:: L U C$ & M5 & Low, N & $2.5 \pm 0.8$ & 13 \\
\hline awf4 & $\mathrm{P}_{W R K Y 29}:: L U C$ & M5 & Low, $\mathrm{N}$ & $3.3 \pm 0.7$ & 21 \\
\hline awf5 & $\mathrm{P}_{W R K Y 29:: L U C}$ & M5 & Low & $9.1 \pm 4.5$ & 19 \\
\hline awf6 & $\mathrm{P}_{W R K Y 29}:: L U C$ & M5 & Low & $46.2 \pm 16.5$ & 21 \\
\hline awf7 & $\mathrm{P}_{W R K Y 29:: L U C}$ & M5 & Low, N & $3.1 \pm 1.1$ & 18 \\
\hline awf8 & $\mathrm{P}_{W R K Y 29}:: L U C$ & M5 & Low & $29.1 \pm 12.3$ & 16 \\
\hline awf & $\mathrm{P}_{\text {WRKY29: }: L U C}$ & M5 & Low, N & $3.2 \pm 1.5$ & 22 \\
\hline awf10 & $\mathrm{P}_{\text {WRKY29: }: L U C}$ & M5 & Low & $26.9 \pm 7.0$ & 22 \\
\hline awf11 & $\mathrm{P}_{W R K Y 29}:: L U C$ & M5 & Low & $9.7 \pm 4.4$ & 21 \\
\hline awf12 & $\mathrm{P}_{\text {WRKY29::LUC }}$ & M5 & Low & $44.4 \pm 20.2$ & 14 \\
\hline awf 13 & $\mathrm{P}_{W R K Y 29}:: L U C$ & M5 & Low & $45.3 \pm 16.5$ & 21 \\
\hline awf14 & $\mathrm{P}_{\text {WRKY29: }: L U C}$ & M5 & Low & $25.0 \pm 10.5$ & 7 \\
\hline awf15 & $\mathrm{P}_{W R K Y 29}:: L U C$ & M5 & Low & $36.3 \pm 15.3$ & 23 \\
\hline awfl6 & $\mathrm{P}_{W R K Y 29}:: L U C$ & M5 & Low & $20.6 \pm 16.9$ & 18 \\
\hline awf 17 & $\mathrm{P}_{W R K Y 29}:: L U C$ & M5 & Low & $21.1 \pm 13.2$ & 9 \\
\hline awf18 & $\mathrm{P}_{\text {WRKY29: }: L U C}$ & M5 & Low, N & $1.8 \pm 0.3$ & 17 \\
\hline awf19 & $\mathrm{P}_{\text {WRKY29: }: L U C}$ & M5 & High & $245.1 \pm 70.7$ & 20 \\
\hline awf 20 & $\mathrm{P}_{W R K Y 29}:: L U C$ & M5 & High & $318.8 \pm 117.4$ & 24 \\
\hline awf 21 & $\mathrm{P}_{W R K Y 29}:: L U C$ & M5 & High, $\mathrm{P}$ & $292.8 \pm 72.6$ & 14 \\
\hline awf 22 & $\mathrm{P}_{W R K Y 29}:: L U C$ & M5 & High & $230.8 \pm 77.0$ & 18 \\
\hline
\end{tabular}

${ }^{a} \mathrm{~N}=$ induction was not observed after treatment and $\mathrm{P}=$ pattern alteration.

b Maximum bioluminescence at peak time. Bioluminescence of mutants are shown as percentages of the wild type (W29-1-4). Values are shown as average \pm standard error. 
flg22 treatment was restored in the complemented transformants (Fig. 7C; Supplementary Fig. S7A). Thus, MutMap successfully identified SNP-8074904 as the causative mutant of the awfl phenotype.
Using such complementation experiments, we also validated the causative mutation in the awf2 mutant (group IV) within THO5 (AT5G42920), a member of the protein family "suppressors of the transcription defects of $h p r l \Delta$ mutants by
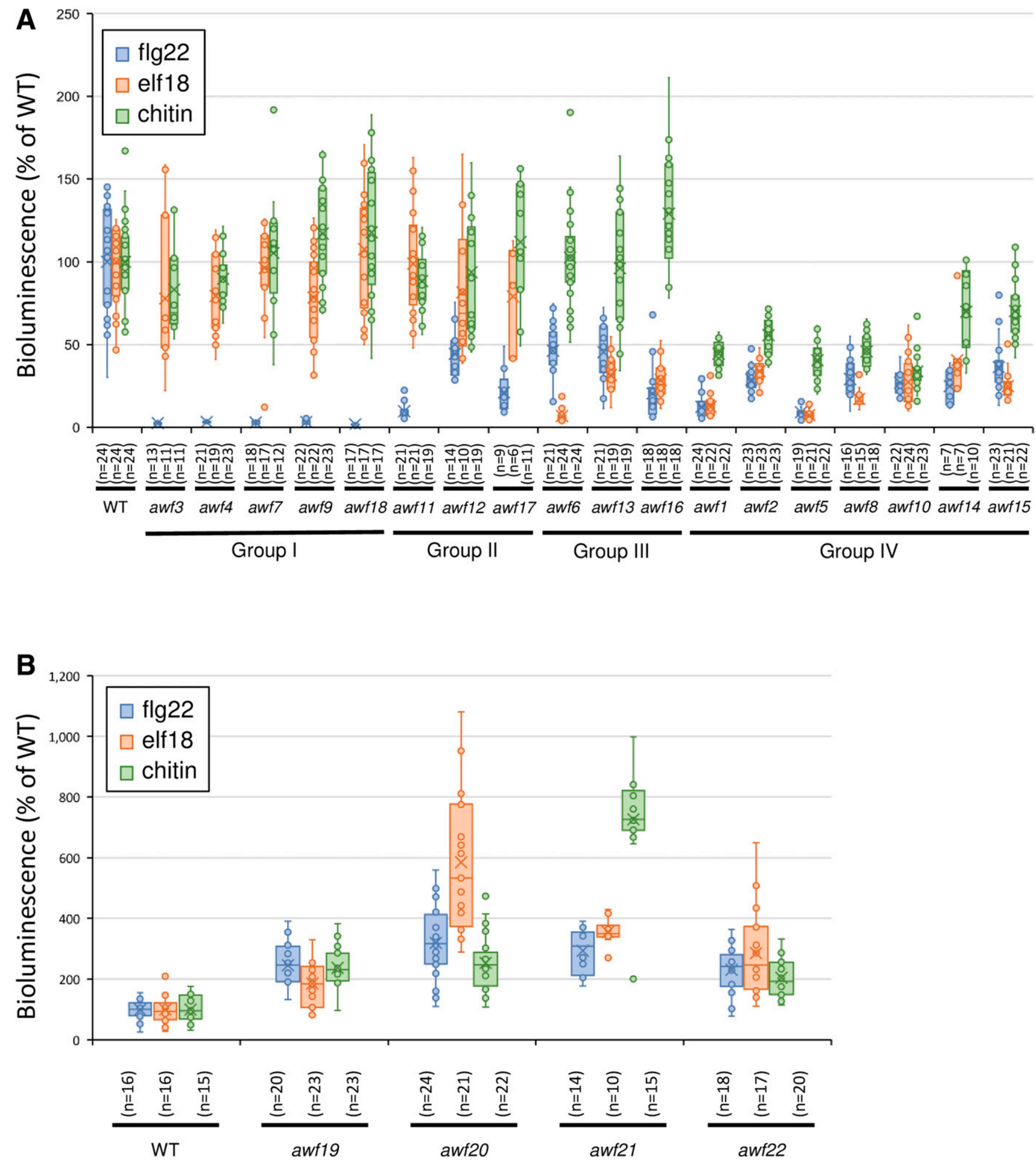

Fig. 4. Classification of mutants with altered WRKY29 expression upon flg22 treatment (awf mutants) by their responses to different pathogen-associated molecular patterns. Eight-day-old mutant seedlings were treated with $0.5 \mu \mathrm{M}$ flg22, $0.5 \mu \mathrm{M}$ elf 18 , or chitin at $1 \mathrm{mg} / \mathrm{ml}$. Response to the three elicitors was monitored with a real-time bioluminescence monitoring system. Bioluminescence at the peak time is shown as percentage of the wild type (W29-1-4) (WT). Data are shown as peak mean \pm standard error from at least seven seedlings per treatment. A, Low-bioluminescence mutants were classified into four groups: group I = mutants with no response to flg22, group II = mutants showing low bioluminescence induction after flg22 treatment, group III = mutants showing low bioluminescence induction after flg22 and elf18 treatment, and group IV = mutants showing low bioluminescence induction after flg22, elf18, and chitin treatment. B, Mutants with increased bioluminescence induction after elicitor treatment. 
overexpression-transcription and export" (THO/TREX) (Fig. 7D to F; Supplementary Fig. S7B), the awf5 mutant (group IV) within CYCLIN-DEPENDENT KINASE 8 (CDK8) (AT5G63610) (Fig. 7G to I; Supplementary Fig. S7C), and the awf16 mutant (group III) within HIGH-LEVEL EXPRESSION OF SUGARINDUCIBLE 2 (HSI2)/VIVIPAROUSI/ABA INSENSITIVE3LIKE1 (VAL1) (AT2G30470) (Fig. 7J to L; Supplementary Fig. S7D). These results demonstrate that applying MutMap to mutants isolated by bioluminescence monitoring successfully identifies causative mutations. MutMap analysis of other awf mutants did not narrow down a single candidate but exhibited multiple candidates; thus, we did not attempt complementation in this study. To further test whether the mutants belonging to the same group (Fig. 4) have mutations in the identical genes, we performed Sanger sequencing of group III mutants (awf6 and awf13) as well as group IV mutants (awf10 and awf15) for the coding regions of ERFO19 (group IV), THO5 (group IV), and HSI2/VAL1 (group III) genes. In these four awf mutants, we could not find any mutation in the three genes, indicating that awf6, awf13, awf10, and awf15 mutants were not caused by mutations in the coding regions of ERFO19, THO5, or HSI2/VAL1.
Gene expression in the selected mutants, awf1, awf2, and awf16.

We selected the three mutants awf1 (erf019-1), awf2 (tho51), and awf16 (hsi2-6) for further characterization. We included awf4 (fls2) as a control and excluded awf5 (cdk8-3) because $C D K 8$ has already been shown to be involved in antibacterial and antifungal defense in Arabidopsis (J. Huang et al. 2019; Zhu et al. 2014). Before proceeding, we confirmed expression of the WRKY29 gene after flg22 treatment in the mutants by qRT-PCR (Fig. 8A; Supplementary Fig. S8) at the 8-day-old seedling stage as used for bioluminescence measurement. In awf1 (erf019-1) and awf16 (hsi2-6), the expression of WRKY29 showed a clear reduction which is in line with the reduction in bioluminescence. However, in the awf2 (tho5-1) mutant, we found that the level of WRKY29 transcript was not altered, which was inconsistent with the results of bioluminescence and qRT-PCR of $L U C$ transcript (Figs. 3 and 8A; Supplementary Fig. S8). We hypothesize that the regulatory elements interacting with the promoter sequence of WRKY29 (-1,931 to -1) (Serrano et al. 2007) used for driving $L U C$ were, indeed, affected by the tho5 mutation. However, it is possible that

A

FLS2

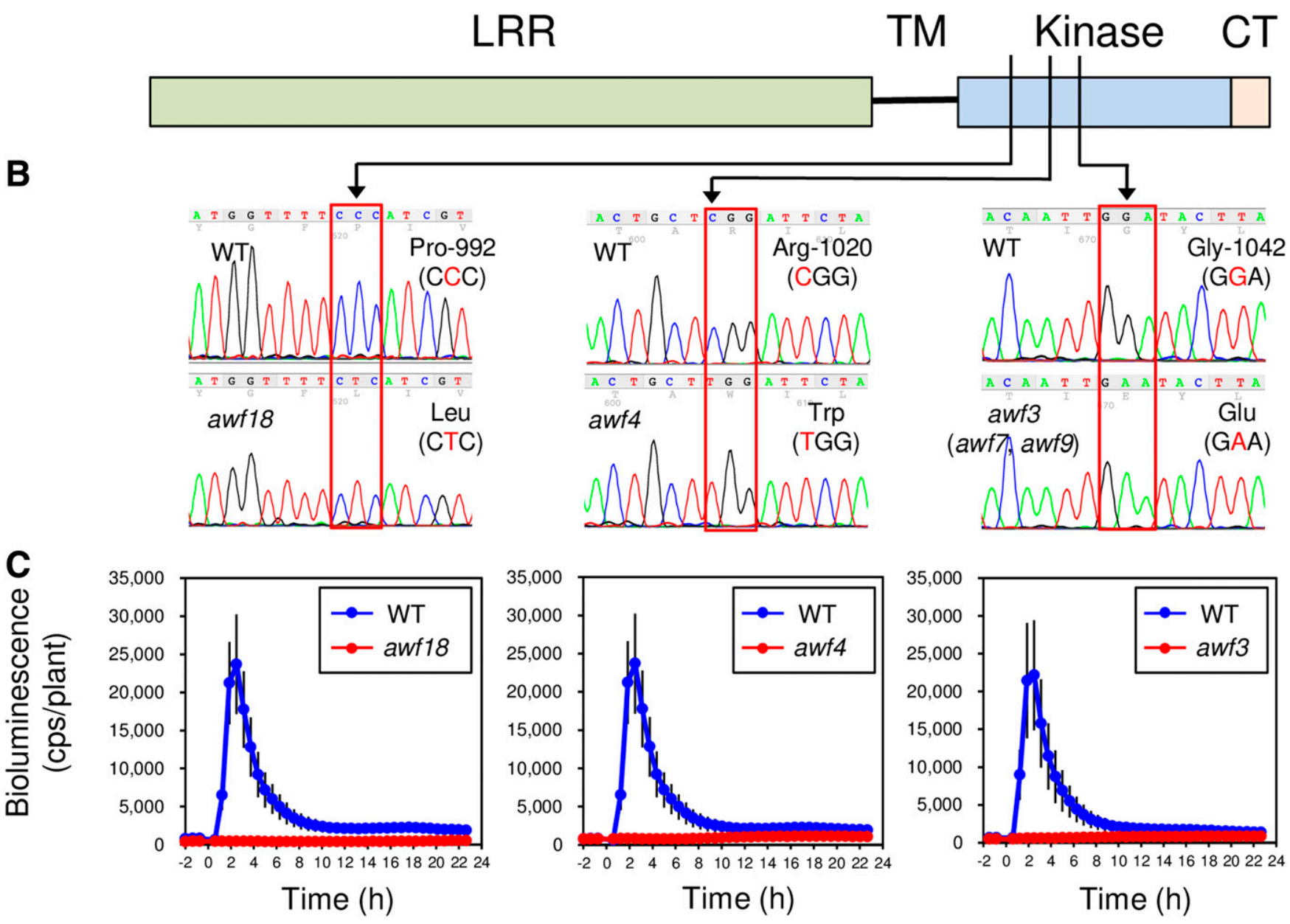

Fig. 5. Mutations in FLS2. A, Domain structure of FLS2 (LRR = leucine rich repeats, $\mathrm{TM}=$ transmembrane, and CT = C-terminal region). B, Confirmation of mutations in FLS2 by Sanger sequencing. Red boxes indicate that mutations in mutants with altered WRKY29 expression upon flg 22 treatment (awf mutants) awf18, awf4, and awf3, awf7, and awf9 have the same mutation as awf3 (G1042E). C, Bioluminescence of mutants with mutations in FLS2. Eight-day-old seedlings of the wild type (W29-1-4) (WT) and mutants were treated with $0.5 \mu \mathrm{M}$ flg22. Bioluminescence from each seedling was monitored with a real-time bioluminescence monitoring system. Data are shown as mean \pm standard error from at least 13 seedlings per treatment. Experiments were conducted three times with similar results. 
additional mechanisms are involved in controlling the endogenous WRKY29 transcript level (see Discussion).

PAMP-induced apoplastic ROS production and WRKY29 gene expression can be uncoupled.

After PAMP perception by PRRs, PTI signaling branches, one pathway led to ROS production and the other to MAPK activation (Segonzac et al. 2011; Xu et al. 2014). The awf mutants were isolated by screening for mutants with alterations in WRKY29 promoter induction, which is reported to be regulated by MAPK signaling (Asai et al. 2002; Eulgem et al. 2000). To test whether these mutants also have alterations in the ROS pathway, we performed a ROS assay by treating leaves with flg22. Genes awf1 (erf019-1), awf2 (tho5-1), and awf16 (hsi2-6) showed ROS generation patterns after flg22 treatment similar to wild-type Col-0 plants and the W29-1-4 reporter line (Supplementary Fig. S9). These results indicate that the mutants tested here have no alternations in the pathway leading to early ROS generation after flg 22 treatment. As expected, awf4 (fls2) did not show flg22-induced ROS generation.

MAPK activity was not affected in awf1, awf2, and awf16.

We also tested activation of MAPK after elicitation of seedlings of awf1 (erf019-1), awf2 (tho5-1), and awf16 (hsi2-6) as well as awf4 (fls2) mutants after flg22 treatment (Supplementary Fig. S10). The awfl awf2, and awfl6 mutants showed awf1
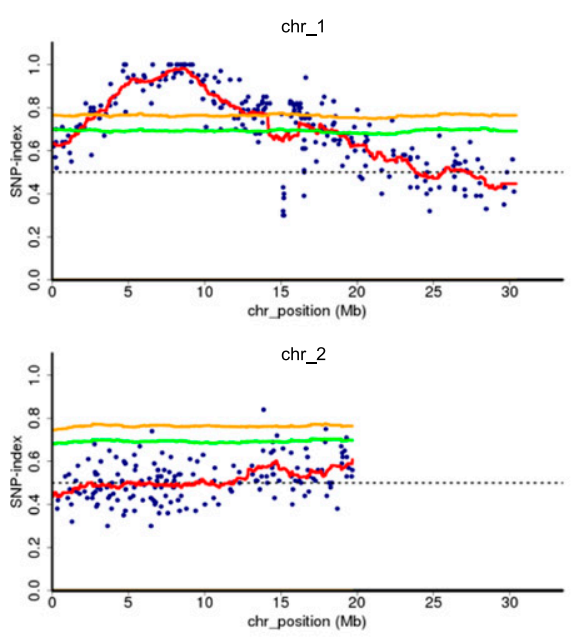

chr_3

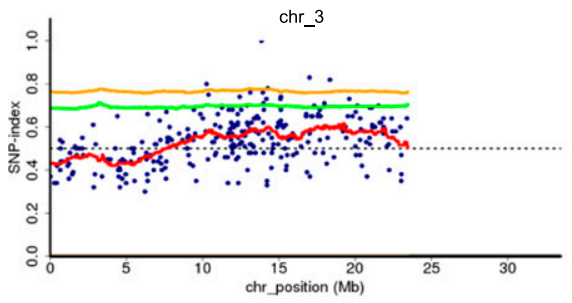

chr_4
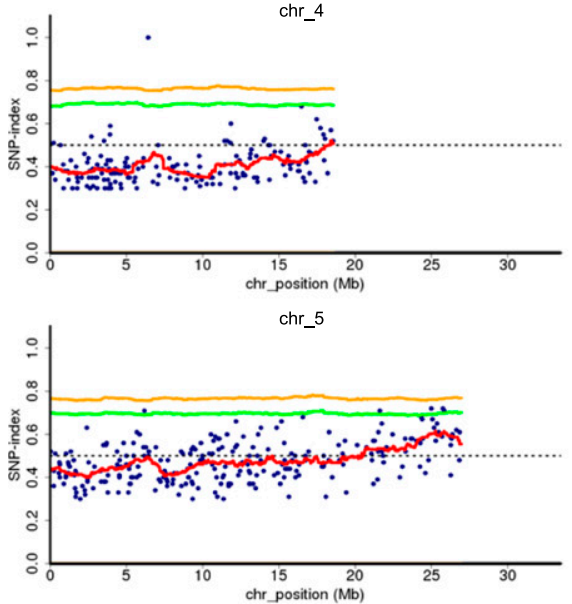

awf2
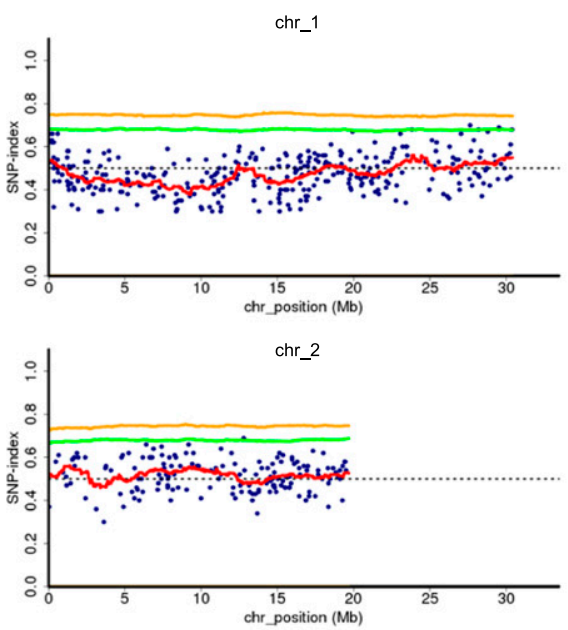

chr_3

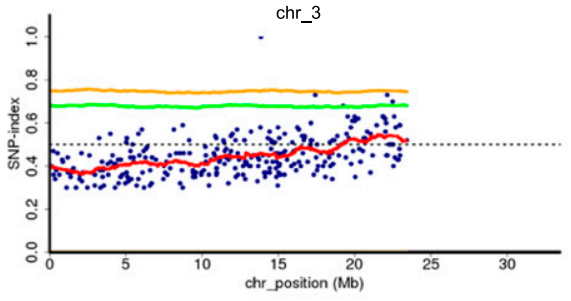

chr_4

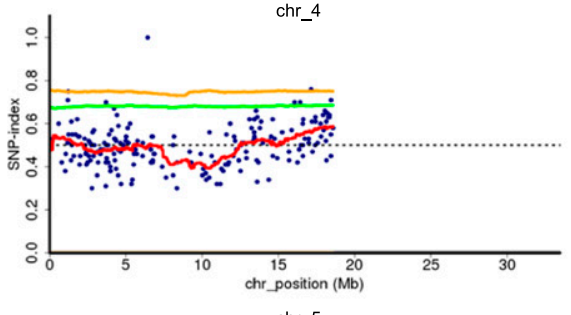

chr_5

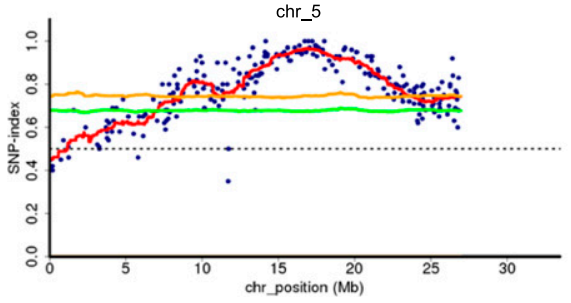

awf9 (fls2)
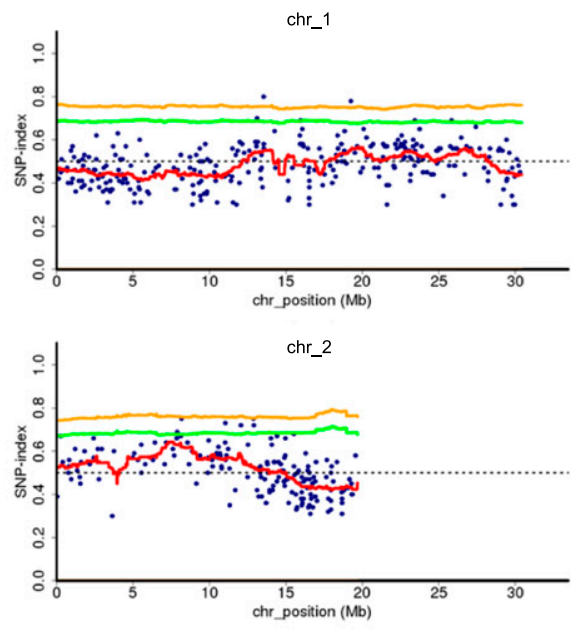

chr_3

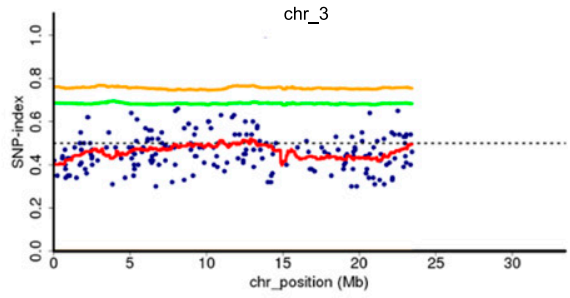

chr_4

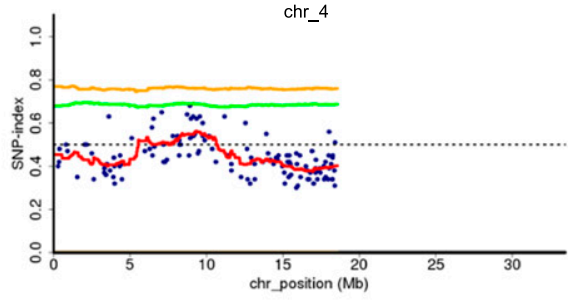

chr_5

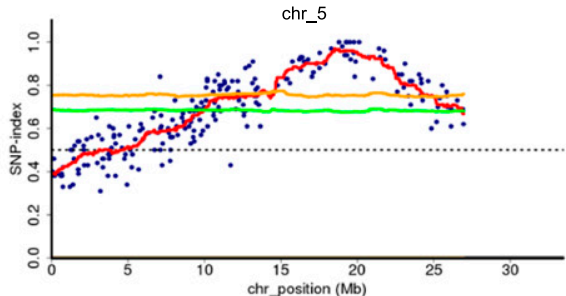

Fig. 6. Application of MutMap to mutants with altered WRKY29 expression upon flg22 treatment (awf mutants) awf1, awf2, and awf9. Single-nucleotide polymorphism (SNP)-index plots of the five chromosomes (chr) of Arabidopsis generated by MutMap analysis, showing a genomic region with the highest SNP-index peak harboring the candidate mutation. Each mutant was crossed to the parental reporter line (W29-1-4) and resulting $\mathrm{F}_{2}$ progeny were tested for their bioluminescence phenotype after flg 22 treatment. Bulked DNA from $30 \mathrm{~F}_{2}$ progeny showing mutant phenotypes were used for sequencing and MutMap analysis. Blue dots correspond to SNPs identified in the mutant lines relative to W29-1-4. The red line represents average SNP-index values across a 2-Mb sliding window with 10-kb increments. Green and yellow lines show the 95 and $99 \%$ confidence limit, respectively, of SNP-index values under the null hypothesis of SNP index $=0.5$. 
A

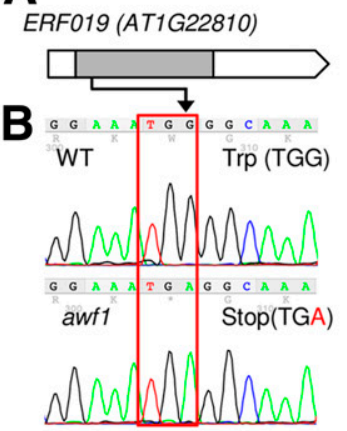

D

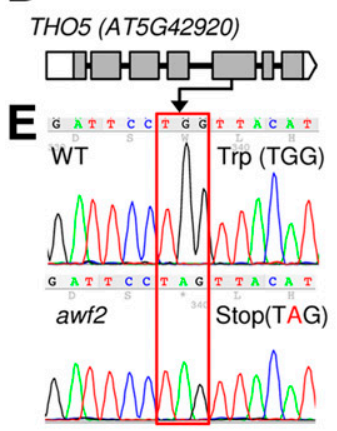

G

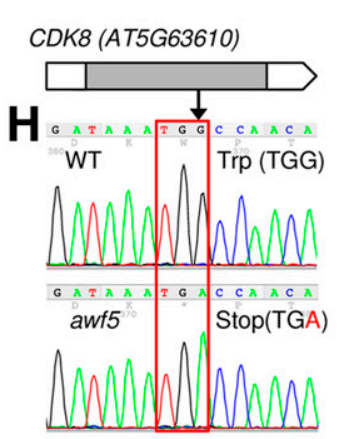

J

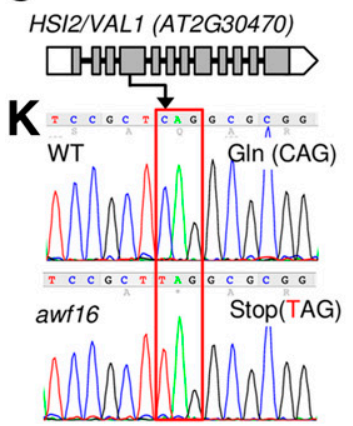

C
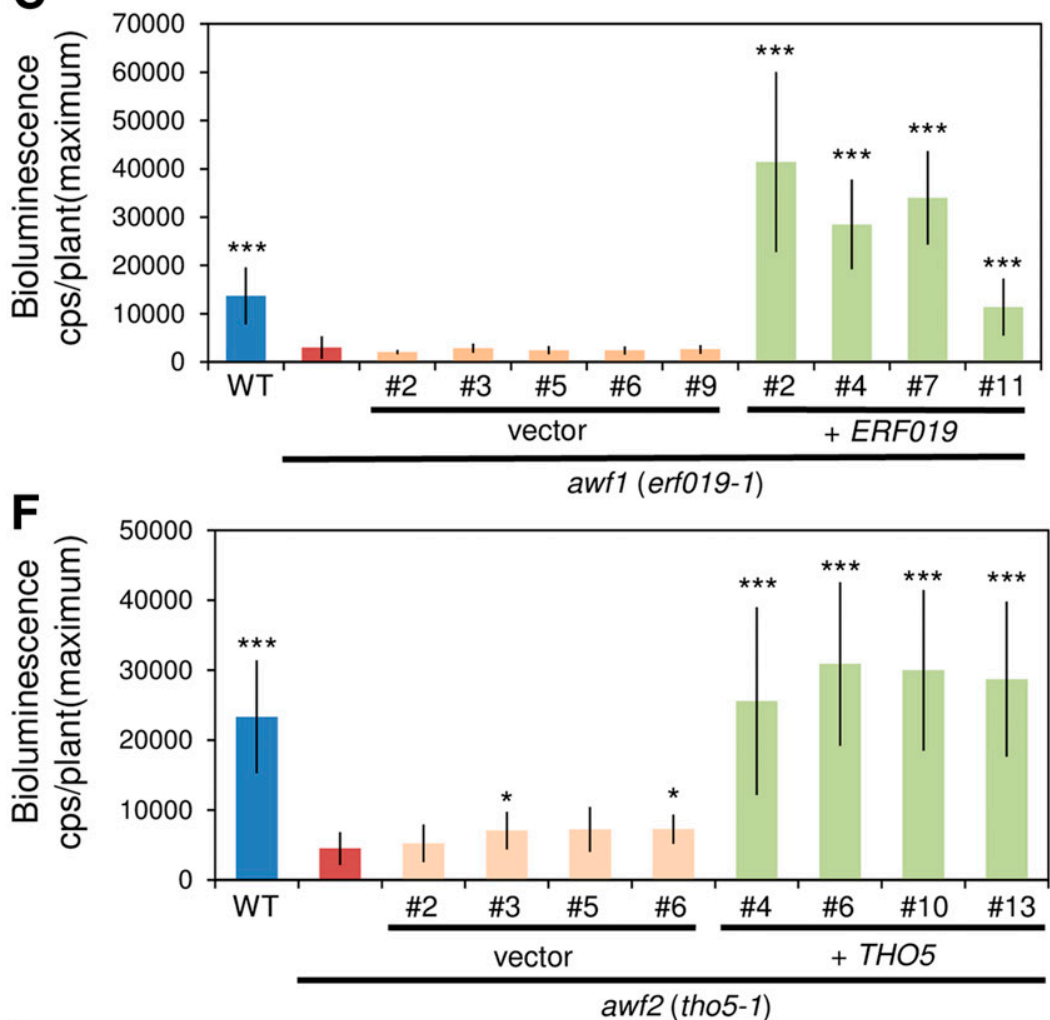

I
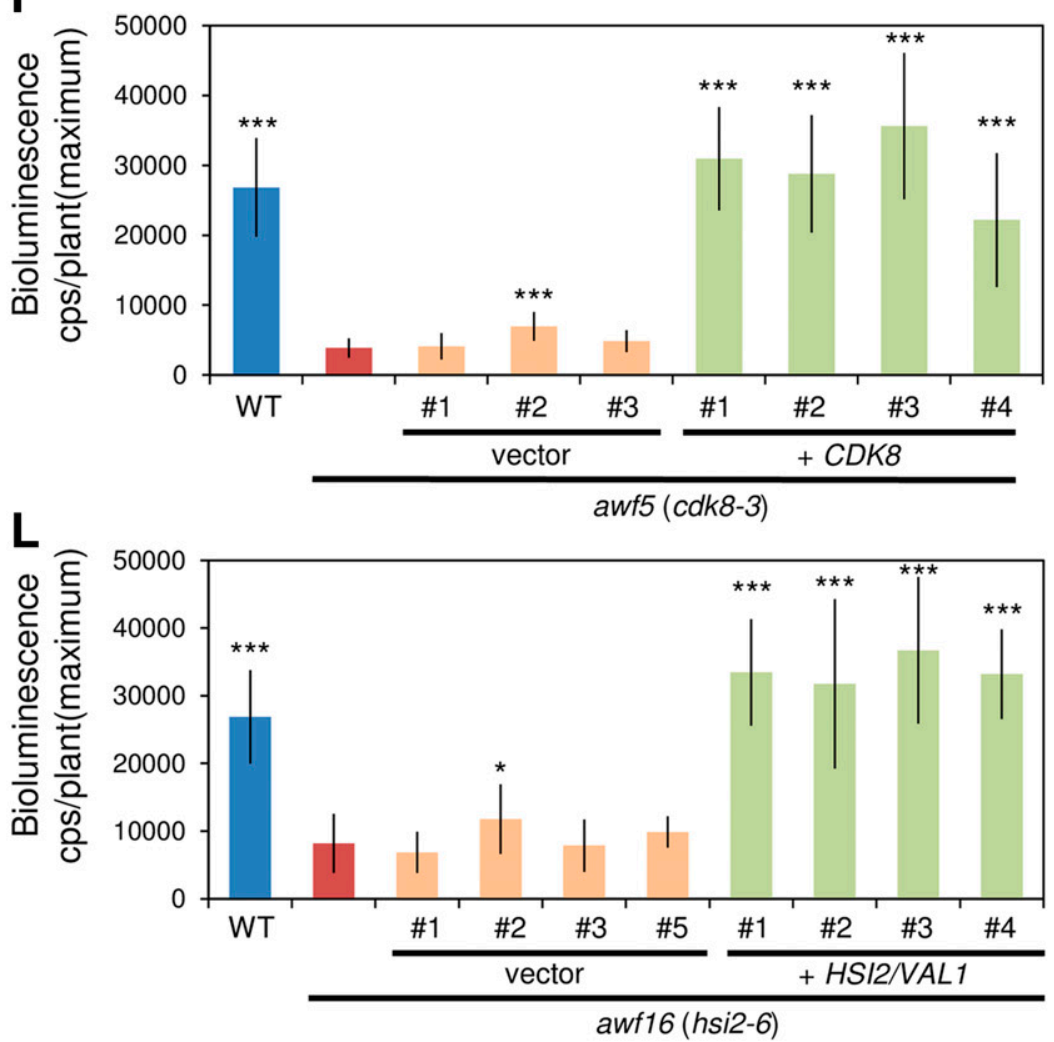

Fig. 7. Genetic complementation of low-bioluminescence mutants. Genetic complementation of mutants with altered WRKY29 expression upon flg22 treatment (awf mutants) A to C, awf1 with ERF019; D to F, awf2 with THO5; G to I, awf5 with CDK8; and $\mathbf{J}$ to L, awf16 with HSI2/VAL1. WT = wild type. Structure of ERF019 (AT1G22810) (A), THO5 (AT5G42920) (D), CDK8 (AT5G63610) (G), and HSI2/VAL1 (AT2G30470) (J) are shown. Open and gray boxes represent untranslated regions and exons, respectively, while lines denote introns. Confirmation of the mutations by Sanger sequencing (B, E, $\mathrm{H}$, and $\mathrm{K})$. Red boxes indicate the mutation sites in $a w f 1(\mathrm{~B}), a w f 2(\mathrm{E}), a w f 5(\mathrm{H})$, and $a w f 16(\mathrm{~K})$. Eight-day-old seedlings of the WT (W29-1-4); the four mutants $a w f 1, a w f 2$, $a w f 5$, and $a w f 16$; as well as mutants transformed with the indicated gene or empty vector (vector) were treated with $0.5 \mu \mathrm{M}$ flg 22 and bioluminescence was monitored with a real-time bioluminescence monitoring system. Data are shown as peak mean \pm standard error from at least five seedlings per line $(\mathrm{C}, \mathrm{F}, \mathrm{I}$, and L). Asterisks indicate significant differences compared with the bioluminescence of the respective $a w f$ mutant $(*$ and $* * *$ indicate $P<0.05$ and 0.001 , respectively; two-tailed $t$ tests). 
MAPK activation, presumably of MPK6 (approximately $47 \mathrm{kDa}$ ), MPK3 (approximately $43 \mathrm{kDa}$ ), and MPK4 (approximately $38 \mathrm{kDa}$ ) (Frei dit Frey et al. 2014; Ranf et al. 2011), similar to the wild-type W29-1-4 reporter, whereas the awf4 (fls2) mutant showed no MAPK activation after flg22 treatment, as expected (Supplementary Fig. S10).

\section{ERF019, THO5, and HSI2 regulate plant immunity.}

To test whether the genes identified in this study indeed regulate Arabidopsis immunity, we challenged 5-week-old plants of the mutants with $P$. syringae pv. tomato DC3000. We found that awf1 (erf019-1), awf2 (tho5-1), and awf16 (hsi2) were more susceptible to $P$. syringae pv. tomato DC3000 than the wild-type W29-1-4 reporter line (Fig. 8B), and their susceptibility was comparable with that of the awf4 (fls2) mutant. These results indicate that the identified genes are involved in the basal resistance against $P$. syringae pv. tomato DC3000. We also tested WRKY29 expression after flg22 treatment in 5week-old plants by qRT-PCR (Supplementary Fig. S11). At 60 min after elicitation, WRKY29 expression was lower in all three awf mutants whereas, at $180 \mathrm{~min}$ after elicitation, there
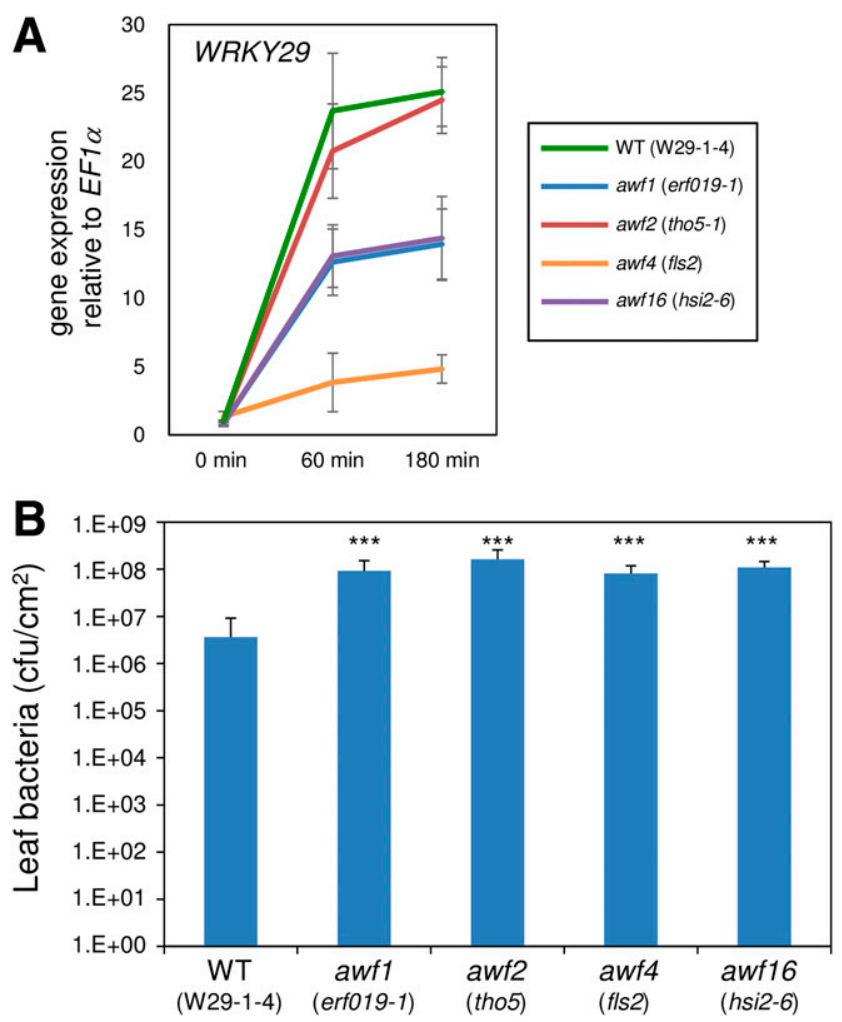

Fig. 8. Pathogen-associated molecular pattern-triggered immunity analysis in mutants with altered WRKY29 expression upon flg22 treatment (awf mutants) awf1, awf2, and awf16. A, Quantitative reverse-transcription PCR analysis of WRKY29 gene expression in awf mutants. Eight-dayold seedlings of the wild type (W29-1-4) (WT), awf1 (erf019-1), awf2 (tho5-1), awf4 (fls2), and awf16 (hsi2-6) were treated with $0.5 \mu \mathrm{M}$ flg22. Gene expression values of WRKY29 are relative to the EF1 $\alpha$ housekeeping gene (At1g07920) and were normalized to the untreated WT seedlings. Values are shown as mean \pm standard error (SE). Experiments were conducted twice with similar results. B, Enhanced susceptibility of awf1, awf2, and awf16 mutants to Pseudomonas syringae pv. tomato DC3000. Five-week-old plants of the WT (W29-1-4), awf1 (erf019-1), awf2 (tho5-1), awf4 (fls2), and awf16 (hsi2-6) were inoculated with $P$. syringae pv. tomato DC3000 (inoculation dose, optical density at $600 \mathrm{~nm}$ $=0.01)$. Bacterial titers were determined 3 days postinoculation. Values are mean $\pm \mathrm{SE}(n=6)$. Asterisks indicate significant differences compared with leaf bacteria of WT (*** indicates $P<0.001$; two-tailed $t$ tests). was no significant WRKY29 downregulation observed for $a w f 2$ (tho5-1) and awf16 (hsi2-6) (Supplementary Fig. S11).

\section{DISCUSSION}

\section{Real-time bioluminescence monitoring for the study of PTI signaling.}

Here, we used Lumi-Map to successfully identify known and novel PTI signaling components. The major advantage of using real-time bioluminescence monitoring for gene expression studies is that it enables high-throughput analysis of gene expression kinetics in a highly sensitive and quantitative manner over longer (approximately 24-h) time periods (Kondo and Ishiura 1994; Onai et al. 2004). By comparing bioluminescence induction patterns, mutants that alter the transcriptional regulation of the reporter gene can be rapidly identified. In previous reports, two aggie mutants were isolated using a $\mathrm{p} F R K 1: L U C$ reporter Arabidopsis strain (Feng et al. 2015; F. Li et al. 2014). In this screen, bioluminescence was observed at only one time point after elicitation using a conventional luminometer, and map-based cloning was employed to identify the causative mutations.

To identify clock genes in diverse organisms, Ishiura and colleagues have developed a series of automated devices for high-throughput real time monitoring of bioluminescence (Kondo and Ishiura 1994; Kondo et al. 1994; Okamoto et al. 2005a,b,c; Onai et al. 2004). Using one of these devices, they succeeded in identifying the gene PHYTOCLOCK1, which is essential for the Arabidopsis circadian clock (Onai and Ishiura 2005; Onai et al. 2004), thereby demonstrating the power of this phenotyping platform. In the current study, we employed the same device (see Materials and Methods) to address PTI signaling.

\section{Identification of novel genes involved in PTI responses.}

We identified ERF019 as a gene that regulates WRKY29 gene expression during flg22, elf18, and chitin PTI responses (awfl in group IV) (Figs. 4 and 7). This gene is also involved in basal resistance to $P$. syringae pv. tomato infection (Fig. 8). It was recently reported that ERF019-overexpressing Arabidopsis also showed increased susceptibility against $P$. syringae pv. tomato infection (P. Y. Huang et al. 2019). In Arabidopsis, ERFs have been implicated in plant defense responses. Knockdown of some ERFs by RNAi enhanced susceptibility to $P$. syringae pv. tomato (Zhang et al. 2015, 2016). ERF6 is phosphorylated by MPK3 and MPK6 during Botrytis cinerea infection (Meng et al. 2013). ERF104 is reported to be involved in the flg22mediated signaling pathway (Bethke et al. 2009). Thus, it is possible that ERFO19 regulates the transcription of WRKYs, including $W R K Y 29$, in the MAPK pathways responding to bacterial infection. However, the erf019 mutant showed normal ROS production and MAPK activation after flg22 elicitation (Supplementary Figs. S9 and S10), suggesting that ERF019 functions downstream of ROS signaling and the MAPK cascade.

From the mutants showing low bioluminescence after flg22 treatment, we identified the $a w f 2$ mutant (group IV) with a defect in the THO5 gene (Figs. 3, 4, 6, and 7). THO5 is also involved in basal resistance against $P$. syringae pv. tomato infection (Fig. 8B). However, our qRT-PCR of endogenous WRKY29 mRNA did not reveal downregulation after flg22 elicitation (Fig. 8A; Supplementary Fig. S8). We hypothesize that this discrepancy of the results may be explained by the promoter sequence used to drive $L U C$ gene, which was a $1.9-\mathrm{kb}$ fragment $(-1,931$ to -1$)$ (Serrano et al. 2007) upstream of the WRKY29 gene start codon. The possible regulatory elements binding to this $1.9-\mathrm{kb}$ fragment may have been affected by 
THO5 (Fig. 3; Supplementary Fig. S8). However, the endogenous WRKY29 gene expression may be regulated by additional cis elements located beyond the $1.9-\mathrm{kb}$ region, and interactors to such elements may have masked the effect of tho5 that was observed with a shorter promoter used to drive $L U C$. THO5 is a member of THO/TREX family, which is involved in mRNA transport and microRNA synthesis (Francisco-Mangilet et al. 2015; Sørensen et al. 2017; Yelina et al. 2010). Therefore, it could be also possible that tho5 mutation may have altered the posttranscriptional regulation of WRKY transcripts. Future work is required to understand this discrepancy. This observation poses a caveat for reporter analysis that we have to use a sufficiently long promoter sequence to drive the $L U C$ gene to faithfully monitor the expression of the target gene.

We also identified a mutation in HSI2/VAL1 as causative for the awfl6 mutant (group III) (Figs. 4 and 7). A mutation in HSI2/VAL1 was previously identified as causative for an Arabidopsis sugar response mutant, where it seems to function as a transcriptional repressor (Tsukagoshi et al. 2005). It also functions as a transcriptional repressor in flowering and drought stress response (Qüesta et al. 2016; Sharma et al. 2013), demonstrating that it plays a role in diverse processes in Arabidopsis. It will be interesting to study whether HSI2/VALI impinges on flg22 and elf18 signaling pathways through transcriptional repression.

The flg22/FLS2 pathway is the best characterized PTI pathway; many genes involved in this pathway have been described. Arabidopsis mutants defective in ROS production following flg22 treatment have been previously studied (Boutrot et al. 2010), resulting in the isolation of the ethylene-signaling protein EIN2 and aspartate oxidase (Boutrot et al. 2010; Macho et al. 2012). However, the genes identified in the current study (ERF019, THO5, and HSI2/VAL1) did not overlap with the genes previously isolated in the flg22/FLS2 pathway, and are not involved in early ROS generation or activation of the MAPK cascade after flg22 perception by FLS2 (Supplementary Figs. S9 and S10). These results indicate that the genes isolated in the current study function downstream of the ROS and MAPK pathways. The reason that we did not recover bakl mutants in our screen may be because we removed mutants with reduced sizes from our consideration. Indeed, most loss-of-function bak1 mutations are known to cause plant size reduction (Chinchilla et al. 2007; Wierzba and Tax 2016).

Here, we developed Lumi-Map to rapidly identifying genes potentially involved in the downstream signaling of PTI (Fig. 1). The advantage of Lumi-Map resides in its ability to monitor real-time gene expression kinetics of the genes. Large-scale application of Lumi-Map could enable the identification of the full repertoire of signaling components linking a cell surface receptor to a reporter gene. For this purpose, we have already generated Arabidopsis LUC reporter lines with promoters from nine genes exhibiting different response patterns to flg22 (Fig. 2) and are going to enlarge the repertoire, which will be freely available to the research community. A subset of reporter lines showed transient induction and repression (WRKY29, WRKY18, $W R K Y 28$, and $R B O H D$ ), whereas others exhibited persistent and complex expression patterns over the time (PAL1, At1g51890, At2g17740, PR4, and ERF1) (Fig. 2). The mutants in the former should be useful to isolate PRR genes and negative regulators of the early signaling cascade. As shown by the identification of $f l s 2$ in our screen using the WRKY29 reporter line, mutants that are nonresponsive to a specific PAMP are likely mutated in the gene encoding the cognate PRR. This result suggests Lumi-Map as a potentially powerful platform to identify novel PRR-encoding genes and their downstream signaling genes using various PAMPs or microbial extracts as input signals. The reporter lines showing persistent and complex expression patterns after elicitation would allow the identification of mutants with various kinetics patterns that may enable us to address how divergent signal inputs are regulated in the downstream signaling network.

\section{MATERIALS AND METHODS}

\section{Plant materials and growth condition.}

Arabidopsis thaliana (ecotype Col-0) seed were used for the construction of all transgenic lines. Surface-sterilized seed of Arabidopsis were incubated at $4^{\circ} \mathrm{C}$ in the dark for 2 days and grown on Murashige and Skoog (MS) medium (Murashige and Skoog 1962) containing $1.5 \%$ (wt/vol) sucrose and $0.8 \%$ (wt/vol) agar at $23^{\circ} \mathrm{C}$ in light. For seed propagation, we grew Arabidopsis plants on MS agar media for 10 to 14 days before transferring to soil at $23^{\circ} \mathrm{C}$ in continuous light from white fluorescent lamps at a light intensity of $57 \mu \mathrm{mol} \mathrm{m} \mathrm{m}^{-2}$.

\section{Molecular cloning.}

We confirmed the nucleotide sequences of all constructs using standard DNA manipulation and sequencing techniques. Primers used in this study are listed in Supplementary Table S7.

The $\mathrm{P}_{\text {WRKY29: }}: L U C$ reporter gene cassette consisted of the Arabidopsis WRKY29 promoter $\left(\mathrm{P}_{\text {WRKY } 29} ;-1,931\right.$ to -1$)$ (Serrano et al. 2007), the coding region of a modified firefly $L U C$ gene $\left(L U C^{+}\right.$; Promega Corp, Madison, WI, U.S.A.), and $\mathrm{T}_{N O S}$. The

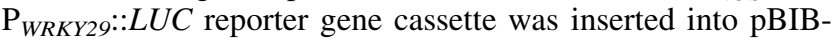
HYG (Becker 1990) with SalI and KpnI. Reporter gene cassettes for eight other reporter lines were constructed using the promoter sequences of the respective genes (Supplementary Table S1).

For complementation of $a w f 1$, a $4.0-\mathrm{kb}$ fragment containing the ERF019 gene and its native promoter was amplified and inserted into pBI101 with BamHI and SacI by In-Fusion HD Cloning Kit (Takara Bio, Shiga, Japan). For awf2, awf5, and awf16, a 5.2-kb fragment containing THO5, a 4.4-kb fragment containing $C D K 8$, and a 6-8 kb fragment containing HSI2/VALI, respectively, were amplified and inserted into pBI101 with SalI and SacI. For awf4, awf9, and awf18, a 5.1-kb fragment containing FLS2 was amplified and inserted into pBI101 with SalI and SacI.

\section{Plant transformation.}

Agrobacterium tumefaciens floral dip was used to transform plants, with minor modifications (Clough and Bent 1998). We used A. tumefaciens strains LBA4404 or GV3101::pMP90 (Koncz and Schell 1986; Weigel and Glazebrook 2002). For constructs in pBIB-HYG, hygromycin B-resistant $T_{1}$ plants were selected and grown using standard techniques. We selected $\mathrm{T}_{2}$ plants that showed a 3:1 segregation ratio for both hygromycin $\mathrm{B}$ resistance and bioluminescence, suggesting that they contained a single T-DNA, and obtained $\mathrm{T}_{3}$ plants from these selected plants. These $\mathrm{T}_{3}$ plants were selected as homozygous by lack of segregation for hygromycin $\mathrm{B}$ resistance and bioluminescence. Bulk $\mathrm{T}_{4}$ seed were generated from the selected $\mathrm{T}_{3}$ plants. For transformation with pBI101-derived vectors, kanamycin was used for selection.

\section{Measurement of bioluminescence response.}

The bioluminescence response of seedlings treated with flg22 was performed as follows. Surface-sterilized seed were incubated at $4^{\circ} \mathrm{C}$ in the dark for 2 days and sown into wells of a 96-well microplate (Luminunc Plates White F96; Thermo Fisher Scientific K.K., Tokyo, Japan) containing $150 \mu$ l of halfstrength liquid MS medium containing $0.5 \%$ sucrose and $50 \mu \mathrm{M}$ D-luciferin-K (Biosynth, Naperville, IL, U.S.A. or Promega Japan, Tokyo, Japan) and germinated in continuous 
light. After 7 days, seedlings were treated with $0.5 \mu \mathrm{M}$ flg22 (GenScript, Piscataway, NJ, U.S.A.), $0.5 \mu \mathrm{M}$ elf18 (Life Technologies Japan Ltd., Tokyo, Japan), or chitin (NA-COS-Y; Yaizu Suisan Kagaku Industry Co., Ltd., Shizuoka, Japan) at $1 \mathrm{mg} / \mathrm{ml}$, and 96-well plates were sealed with a plate seal (Excel Scientific, Victorville, CA, U.S.A. or Plate Seal T, Sanplatec Corp., Osaka, Japan) instead of a plastic cover.

Bioluminescence from each well was measured automatically using a commercially available automated bioluminescence monitoring system (model CL96-4; Churitsu Electric Corp., Nagoya, Aichi, Japan) with a robotic plate conveyor (model CI08L; Churitsu Electric Corp.). Bioluminescence data were analyzed using commercially available software (SL00-01; Churitsu Electric Corp.).

\section{Mutagenesis of W29-1-4 reporter line and mutant screening.}

Approximately 8,000 $\mathrm{T}_{4}$ seeds from the W29-1-4 reporter line were mutagenized by treatment with $0.3 \%$ (vol/vol) EMS (Merck $\mathrm{KGaA}$, Frankfurter, Darmstadt, Germany) for $15 \mathrm{~h}$ at $25^{\circ} \mathrm{C} . \mathrm{M}_{2}$ seed were collected and grouped into six pools, each of which contained seed from approximately 1,300 $\mathrm{M}_{1}$ plants. Approximately 4,000 seedlings from each $\mathbf{M}_{2}$ pool were screened for an altered bioluminescence response following flg22 treatment.

WGS of nine awf mutant lines (Supplementary Table S8) showed that our EMS mutagenesis resulted in 1,000 to 1,500 nucleotide substitutions incorporated per line. If we extrapolate 1,000 SNPs per line to the total 24,000 lines screened, we expect 24,000,000 SNPs in the 24,000 lines. In the arbitrary 1$\mathrm{kb}$ region of the Arabidopsis genome $(135 \mathrm{Mb})$, we expect approximately $178 \mathrm{SNPs}$, on average, after screening all of the lines $(178=24,000,000[135 \mathrm{Mb} / 1 \mathrm{~kb}])$. This figure suggests that the majority of the genes of Arabidopsis genome has been mutated by our condition of EMS mutagenesis.

In $\mathrm{M}_{2}$ screening, we assayed the bioluminescence response from seedlings treated with $0.5 \mu \mathrm{M}$ flg22 using a highthroughput real-time bioluminescence monitoring system (Churitsu Electric Corp.) equipped with Electron Multiplying Charge-Coupled Device image sensors (Andor Japan, Tokyo, Japan), essentially as described by Kondo and Ishiura (1994).

\section{Generation of $F_{2}$ progeny and WGS.}

To generate the $F_{2}$ progeny used for bulk sequencing, each mutant was crossed to the W29-1-4 reporter line (the parental line of the mutants) and the resulting $F_{1}$ progeny were selfpollinated to produce $F_{2}$ seed. Bulk DNA for MutMap analysis was prepared from equal amounts of $30 \mathrm{~F}_{2}$ mutant individuals. For WGS, DNA samples were extracted from young leaves with the DNeasy Plant Mini Kit (Qiagen K.K., Tokyo, Japan). We prepared 11 sequence libraries for WGS: the WRKY29 reporter line T3_W29-1-4 and awf3 (read length; 75 bp, paired-end sequencing [PE]) and awf1, awf2, awf5, awf8, awf9, awf14, awf16, awf 19, and awf 21 mutant lines (150 bp, PE). Sequence libraries for PE short reads were constructed using an Illumina TruSeq DNA LT Sample Prep Kit (Illumina K.K., Tokyo, Japan). The libraries were sequenced on a HiSeq high-output (150 bp, PE), NextSeq500 (75 bp, PE), or MiSeq (75 bp, PE). WGS data has been deposited with the DNA Data Bank of Japan BioProject under PRJDB6767. WGS of the 10 independent mutants indicated that each line had 1,470 \pm 266 (mean \pm standard deviation; range 944 to 1,795) SNPs relative to the W29-1-4, the wild-type reporter strain (Supplementary Table S8).

\section{MutMap analysis.}

The W29-1-4 reference sequence was constructed by replacing nucleotides in Col-0 with those of W29-1-4 at the 581 SNP positions identified by aligning $3.4 \mathrm{~Gb}$ of Illumina W29-1-4 short reads to the Col-0 reference genome (EnsemblePlants Arabidopsis thaliana TAIR10 release-36), as previously described (Takagi et al. 2015).

Illumina short reads generated from the bulked DNA samples were filtered by Phred quality score and aligned to the W29-1-4 reference sequence using BWA (Li and Durbin 2009). Alignments were converted to SAM/BAM files using SAMtools $(\mathrm{H}$. Li et al. 2009), and low-quality SNPs were excluded with a Coval filter (Kosugi et al. 2013). Additionally, SNPs that were detected by selfalignment of the parental W29-1-4 short reads to the W29-1-4 reference sequence were judged to be spurious and were excluded from the analysis. SNP index was calculated at all SNP positions with Coval (Kosugi et al. 2013). All steps were automatically processed using the MutMap_v1.4.5 pipeline. The mutation responsible to the given phenotype should have SNP index $=1$. However, sometimes NGS short reads contain sequence errors and they may cause SNP index $<1$. Also, there is a possibility that wild-type $\mathrm{F}_{2}$ plants were erroneously included in the mutant bulk, which may cause SNP index $<1$. Therefore, we sought the causative SNPs from the SNPs showing SNP index being close to 1 .

\section{RNA extraction and qRT-PCR.}

Gene expression assays were performed on 8-day-old seedlings and 5-week-old mature leaves. Seedlings and leaves were treated with $0.5 \mu \mathrm{M}$ flg 22 for 60 or $180 \mathrm{~min}$ and frozen in liquid nitrogen. Total RNA was extracted using the RNeasy Plant Mini kit (Qiagen K.K.) according to the manufacturer's instructions. The RNA samples were treated with TURBO DNase (Thermo Fisher Scientific K.K.). cDNA was synthesized using ReverTra Ace (Toyobo, Osaka, Japan). The qRT-PCR was performed as previously described by Kobayashi et al. (2017). Gene EFl $\alpha$ (Atlg07920) was used as internal control. Primers used for

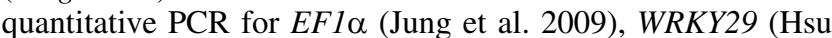
et al. 2013), FRK1 (He et al. 2006), CYP81F2 (At5g57220), and $L U C$ are listed in Supplementary Table S7.

\section{Bacterial inoculation assay.}

Inoculation was performed as previously described by Jelenska et al. (2010), Yamada et al. (2016), and Zhang et al. (2017), with some modifications.

Briefly, P. syringae pv. tomato DC3000 was preincubated in King's B (KB) liquid medium with rifampicin at $50 \mu \mathrm{g} / \mathrm{ml}$ at $28^{\circ} \mathrm{C}$ overnight and incubated 4 to $6 \mathrm{~h}$ at $28^{\circ} \mathrm{C}$. Freshly grown bacteria from $\mathrm{KB}$ liquid medium with $50 \mu \mathrm{g}$ of rifampicin per milliliter were used to prepare the inoculum. After washing with $10 \mathrm{mM} \mathrm{MgSO}_{4}$, the inoculum was resuspended in $10 \mathrm{mM}$ $\mathrm{MgSO}_{4}$ containing $0.01 \%$ (vol/vol) Silwet L-77 to a final optical density at $600 \mathrm{~nm}=0.01$ and subsequently sprayed on 5-weekold Arabidopsis. Inoculated plants were kept in a box covered with plastic for 3 days. Two leaf discs from four or five independent plants were pooled and serially diluted with $10 \mathrm{mM}$ $\mathrm{MgSO}_{4}$. Each dilution was plated on Luria-Bertani agar medium containing antibiotics and incubated at $28^{\circ} \mathrm{C}$ for 2 days.

\section{ACKNOWLEDGMENTS}

We thank K. Ito, A. Uemura, E. Kanzaki, E. Sato, and H. Kikuchi (IBRC) for technical assistance. Computational analysis was partially performed on the NIG supercomputer at the ROIS National Institute of Genetics.

\section{AUTHOR-RECOMMENDED INTERNET RESOURCES}

\footnotetext{
Churitsu Electric Corp.:

https://www.churitsu.co.jp/products/bio/highthroughput.html

EnsemplePlants Arabidopsis thaliana (TAIR10):

https://plants.ensembl.org/Arabidopsis_thaliana/Info/Index

MutMap_v1.4.5 pipeline:

http://genome-e.ibrc.or.jp/home/bioinformatics-team/mutmap
} 


\section{LITERATURE CITED}

Abe, A., Kosugi, S., Yoshida, K., Natsume, S., Takagi, H., Kanzaki, H., Matsumura, H., Yoshida, K., Mitsuoka, C., Tamiru, M., Innan, H., Cano, L., Kamoun, S., and Terauchi, R. 2012. Genome sequencing reveals agronomically important loci in rice using MutMap. Nat. Biotechnol. 30: 174-178.

Asai, T., Tena, G., Plotnikova, J., Willmann, M. R., Chiu, W. L., GómezGómez, L., Boller, T., Ausubel, F. M., and Sheen, J. 2002. MAP kinase signalling cascade in Arabidopsis innate immunity. Nature 415: 977-983.

Becker, D. 1990. Binary vectors which allow the exchange of plant selectable markers and reporter genes. Nucleic Acids Res. 18:203.

Bethke, G., Unthan, T., Uhrig, J. F., Pöschl, Y., Gust, A. A., Scheel, D., and Lee, J. 2009. Flg22 regulates the release of an ethylene response factor substrate from MAP kinase 6 in Arabidopsis thaliana via ethylene signaling. Proc. Natl. Acad. Sci. U.S.A. 106:8067-8072.

Birkenbihl, R. P., Liu, S., and Somssich, I. E. 2017. Transcriptional events defining plant immune responses. Curr. Opin. Plant Biol. 38:1-9.

Boller, T., and Felix, G. 2009. A renaissance of elicitors: Perception of microbe-associated molecular patterns and danger signals by patternrecognition receptors. Annu. Rev. Plant Biol. 60:379-406.

Boudsocq, M., Willmann, M. R., McCormack, M., Lee, H., Shan, L., He, P., Bush, J., Cheng, S. H., and Sheen, J. 2010. Differential innate immune signalling via $\mathrm{Ca}^{2+}$ sensor protein kinases. Nature 464:418-422.

Boutrot, F., Segonzac, C., Chang, K. N., Qiao, H., Ecker, J. R., Zipfel, C., and Rathjen, J. P. 2010. Direct transcriptional control of the Arabidopsis immune receptor FLS2 by the ethylene-dependent transcription factors EIN3 and EIL1. Proc. Natl. Acad. Sci. U.S.A. 107:14502-14507.

Boutrot, F., and Zipfel, C. 2017. Function, discovery, and exploitation of plant pattern recognition receptors for broad-spectrum disease resistance. Annu. Rev. Phytopathol. 55:257-286.

Cao, Y., Liang, Y., Tanaka, K., Nguyen, C. T., Jedrzejczak, R. P., Joachimiak, A., and Stacey, G. 2014. The kinase LYK5 is a major chitin receptor in Arabidopsis and forms a chitin-induced complex with related kinase CERK1. eLife 3:e03766.

Chinchilla, D., Zipfel, C., Robatzek, S., Kemmerling, B., Nürnberger, T., Jones, J. D., Felix, G., and Boller, T. 2007. A flagellin-induced complex of the receptor FLS2 and BAK1 initiates plant defence. Nature 448: 497-500.

Clough, S. J., and Bent, A. F. 1998. Floral dip: A simplified method for Agrobacterium-mediated transformation of Arabidopsis thaliana. Plant J. 16:735-743.

Denoux, C., Galletti, R., Mammarella, N., Gopalan, S., Werck, D., De Lorenzo, G., Ferrari, S., Ausubel, F. M., and Dewdney, J. 2008. Activation of defense response pathways by OGs and Flg22 elicitors in Arabidopsis seedlings. Mol. Plant 1:423-445.

Dubiella, U., Seybold, H., Durian, G., Komander, E., Lassig, R., Witte, C. P., Schulze, W. X., and Romeis, T. 2013. Calcium-dependent protein kinase/NADPH oxidase activation circuit is required for rapid defense signal propagation. Proc. Natl. Acad. Sci. U.S.A. 110:8744-8749.

Eulgem, T., Rushton, P. J., Robatzek, S., and Somssich, I. E. 2000. The WRKY superfamily of plant transcription factors. Trends Plant Sci. 5: 199-206.

Felix, G., Duran, J. D., Volko, S., and Boller, T. 1999. Plants have a sensitive perception system for the most conserved domain of bacterial flagellin. Plant J. 18:265-276.

Feng, B., Liu, C., de Oliveira, M. V., Intorne, A. C., Li, B., Babilonia, K., de Souza Filho, G. A., Shan, L., and He, P. 2015. Protein poly(ADP-ribosyl) ation regulates Arabidopsis immune gene expression and defense responses. PLoS Genet. 11:e1004936.

Francisco-Mangilet, A. G., Karlsson, P., Kim, M. H., Eo, H. J., Oh, S. A., Kim, J. H., Kulcheski, F. R., Park, S. K., and Manavella, P. A. 2015. $\mathrm{THO} 2$, a core member of the THO/TREX complex, is required for microRNA production in Arabidopsis. Plant J. 82:1018-1029.

Frei dit Frey, N., Garcia, A. V., Bigeard, J., Zaag, R., Bueso, E., Garmier, M., Pateyron, S., de Tauzia-Moreau, M. L., Brunaud, V., Balzergue, S., Colcombet, J., Aubourg, S., Martin-Magniette, M. L., and Hirt, H. 2014. Functional analysis of Arabidopsis immune-related MAPKs uncovers a role for MPK3 as negative regulator of inducible defences. Genome Biol. 15:R87.

Gómez-Gómez, L., and Boller, T. 2000. FLS2: An LRR receptor-like kinase involved in the perception of the bacterial elicitor flagellin in Arabidopsis. Mol. Cell 5:1003-1011.

He, P., Shan, L., Lin, N. C., Martin, G. B., Kemmerling, B., Nürnberger, T., and Sheen, J. 2006. Specific bacterial suppressors of MAMP signaling upstream of MAPKKK in Arabidopsis innate immunity. Cell 125: 563-575.
Heese, A., Hann, D. R., Gimenez-Ibanez, S., Jones, A. M., He, K., Li, J., Schroeder, J. I., Peck, S. C., and Rathjen, J. P. 2007. The receptor-like kinase SERK3/BAK1 is a central regulator of innate immunity in plants. Proc. Natl. Acad. Sci. U.S.A. 104:12217-12222.

Hsu, F. C., Chou, M. Y., Chou, S. J., Li, Y. R., Peng, H. P., and Shih, M. C. 2013. Submergence confers immunity mediated by the WRKY22 transcription factor in Arabidopsis. Plant Cell 25:2699-2713.

Huang, J., Sun, Y., Orduna, A. R., Jetter, R., and Li, X. 2019. The Mediator kinase module serves as a positive regulator of salicylic acid accumulation and systemic acquired resistance. Plant J. 98:842-852.

Huang, P. Y., Zhang, J., Jiang, B., Chan, C., Yu, J. H., Lu, Y. P., Chung, K., and Zimmerli, L. 2019. NINJA-associated ERF19 negatively regulates Arabidopsis pattern-triggered immunity. J. Exp. Bot. 70:1033-1047.

Jelenska, J., van Hal, J. A., and Greenberg, J. T. 2010. Pseudomonas syringae hijacks plant stress chaperone machinery for virulence. Proc. Natl. Acad. Sci. U.S.A. 107:13177-13182.

Jung, H. W., Tschaplinski, T. J., Wang, L., Glazebrook, J., and Greenberg, J. T. 2009. Priming in systemic plant immunity. Science 324:89-91.

Kadota, Y., Sklenar, J., Derbyshire, P., Stransfeld, L., Asai, S., Ntoukakis, V., Jones, J. D., Shirasu, K., Menke, F., Jones, A., and Zipfel, C. 2014 Direct regulation of the NADPH oxidase RBOHD by the PRRassociated kinase BIK1 during plant immunity. Mol. Cell 54:43-55.

Kobayashi, M., Hiraka, Y., Abe, A., Yaegashi, H., Natsume, S., Kikuchi, H., Takagi, H., Saitoh, H., Win, J., Kamoun, S., and Terauchi, R. 2017. Genome analysis of the foxtail millet pathogen Sclerospora graminicola reveals the complex effector repertoire of graminicolous downy mildews. BMC Genomics 18:897.

Koncz, G. R., and Schell, J. 1986. The promoter of the $\mathrm{T}_{\mathrm{L}}$-DNA gene 5 controls the tissue-specific expression of chimeric genes carried by a novel type of Agrobacterium binary vector. Mol. Gen. Genet. 204: 383-396.

Kondo, T., and Ishiura, M. 1994. Circadian rhythms of cyanobacteria: Monitoring the biological clocks of individual colonies by bioluminescence. J. Bacteriol. 176:1881-1885.

Kondo, T., Tsinoremas, N. F., Golden, S. S., Johnson, C. H., Kutsuna, S., and Ishiura, M. 1994. Circadian clock mutants of cyanobacteria. Science 266:1233-1236.

Kosugi, S., Natsume, S., Yoshida, K., MacLean, D., Cano, L., Kamoun, S., and Terauchi, R. 2013. Coval: Improving alignment quality and variant calling accuracy for next-generation sequencing data. PLoS One 8: e75402.

Li, F., Cheng, C., Cui, F., de Oliveira, M. V., Yu, X., Meng, X., Intorne, A. C., Babilonia, K., Li, M., Li, B., Chen, S., Ma, X., Xiao, S., Zheng, Y., Fei, Z., Metz, R. P., Johnson, C. D., Koiwa, H., Sun, W., Li, Z., de Souza Filho, G. A., Shan, L., and He, P. 2014. Modulation of RNA polymerase II phosphorylation downstream of pathogen perception orchestrates plant immunity. Cell Host Microbe 16:748-758.

Li, H., and Durbin, R. 2009. Fast and accurate short read alignment with Burrows-Wheeler transform. Bioinformatics 25:1754-1760.

Li, H., Handsaker, B., Wysoker, A., Fennell, T., Ruan, J., Homer, N., Marth, G., Abecasis, G., and Durbin, R.; 1000 Genome Project Data Processing Subgroup. 2009. The Sequence Alignment/Map format and SAMtools. Bioinformatics 25:2078-2079.

Li, J., Zhao-Hui, C., Batoux, M., Nekrasov, V., Roux, M., Chinchilla, D., Zipfel, C., and Jones, J. D. 2009. Specific ER quality control components required for biogenesis of the plant innate immune receptor EFR. Proc. Natl. Acad. Sci. U.S.A. 106:15973-15978.

Li, L., Li, M., Yu, L., Zhou, Z., Liang, X., Liu, Z., Cai, G., Gao, L., Zhang, X., Wang, Y., Chen, S., and Zhou, J. M. 2014. The FLS2-associated kinase BIK1 directly phosphorylates the NADPH oxidase RbohD to control plant immunity. Cell Host Microbe 15:329-338.

Lu, D., Wu, S., Gao, X., Zhang, Y., Shan, L., and He, P. 2010. A receptorlike cytoplasmic kinase, BIK1, associates with a flagellin receptor complex to initiate plant innate immunity. Proc. Natl. Acad. Sci. U.S.A. 107:496-501.

Lu, X., Tintor, N., Mentzel, T., Kombrink, E., Boller, T., Robatzek, S., Schulze-Lefert, P., and Saijo, Y. 2009. Uncoupling of sustained MAMP receptor signaling from early outputs in an Arabidopsis endoplasmic reticulum glucosidase II allele. Proc. Natl. Acad. Sci. U.S.A. 106: 22522-22527.

Macho, A. P., Boutrot, F., Rathjen, J. P., and Zipfel, C. 2012. Aspartate oxidase plays an important role in Arabidopsis stomatal immunity. Plant Physiol. 159:1845-1856.

Meng, X., Xu, J., He, Y., Yang, K. Y., Mordorski, B., Liu, Y., and Zhang, S. 2013. Phosphorylation of an ERF transcription factor by Arabidopsis MPK3/MPK6 regulates plant defense gene induction and fungal resistance. Plant Cell 25:1126-1142. 
Miya, A., Albert, P., Shinya, T., Desaki, Y., Ichimura, K., Shirasu, K. Narusaka, Y., Kawakami, N., Kaku, H., and Shibuya, N. 2007. CERK1, a LysM receptor kinase, is essential for chitin elicitor signaling in Arabidopsis. Proc. Natl. Acad. Sci. U.S.A. 104:19613-19618.

Monaghan, J., Matschi, S., Shorinola, O., Rovenich, H., Matei, A., Segonzac, C., Malinovsky, F. G., Rathjen, J. P., MacLean, D., Romeis, T., and Zipfel, C. 2014. The calcium-dependent protein kinase CPK28 buffers plant immunity and regulates BIK1 turnover. Cell Host Microbe 16:605-615

Murashige, T., and Skoog, F. 1962. A revised medium for rapid growth and bioassays with tobacco tissue cultures. Physiol. Plant. 15:473-497.

Nakano, T., Suzuki, K., Fujimura, T., and Shinshi, H. 2006. Genome-wide analysis of the ERF gene family in Arabidopsis and rice. Plant Physiol. 140:411-432.

Navarro, L., Zipfel, C., Rowland, O., Keller, I., Robatzek, S., Boller, T., and Jones, J. D. 2004. The transcriptional innate immune response to flg22. Interplay and overlap with Avr gene-dependent defense responses and bacterial pathogenesis. Plant Physiol. 135:1113-1128.

Nekrasov, V., Li, J., Batoux, M., Roux, M., Chu, Z. H., Lacombe, S., Rougon, A., Bittel, P., Kiss-Papp, M., Chinchilla, D., van Esse, H. P., Jorda, L., Schwessinger, B., Nicaise, V., Thomma, B. P., Molina, A., Jones, J. D., and Zipfel, C. 2009. Control of the pattern-recognition receptor EFR by an ER protein complex in plant immunity. EMBO J. 28:3428-3438.

Okamoto, K., Onai, K., Ezaki, N., Ofuchi, T., and Ishiura, M. 2005a. An automated apparatus for the real-time monitoring of bioluminescence in plants. Anal. Biochem. 340:187-192.

Okamoto, K., Onai, K., Furusawa, T., and Ishiura, M. 2005b. A portable integrated automatic apparatus for the real-time monitoring of bioluminescence in plants. Plant Cell Environ. 28:1305-1315.

Okamoto, K., Onai, K., and Ishiura, M. 2005c. RAP, an integrated program for monitoring bioluminescence and analyzing circadian rhythms in real time. Anal. Biochem. 340:193-200.

Onai, K., and Ishiura, M. 2005. PHYTOCLOCK 1 encoding a novel GARP protein essential for the Arabidopsis circadian clock. Genes Cells 10:963-972.

Onai, K., Okamoto, K., Nishimoto, H., Morioka, C., Hirano, M., Kami-Ike, N., and Ishiura, M. 2004. Large-scale screening of Arabidopsis circadian clock mutants by a high-throughput real-time bioluminescence monitoring system. Plant J. 40:1-11.

Qüesta, J. I., Song, J., Geraldo, N., An, H., and Dean, C. 2016. Arabidopsis transcriptional repressor VAL1 triggers Polycomb silencing at FLC during vernalization. Science 353:485-488.

Ranf, S., Eschen-Lippold, L., Pecher, P., Lee, J., and Scheel, D. 2011. Interplay between calcium signalling and early signalling elements during defence responses to microbe- or damage-associated molecular patterns. Plant J. 68:100-113.

Ranf, S., Grimmer, J., Pöschl, Y., Pecher, P., Chinchilla, D., Scheel, D., and Lee, J. 2012. Defense-related calcium signaling mutants uncovered via a quantitative high-throughput screen in Arabidopsis thaliana. Mol. Plant 5:115-130.

Rasmussen, M. W., Roux, M., Petersen, M., and Mundy, J. 2012. MAP kinase cascades in Arabidopsis innate immunity. Front. Plant Sci. 3:169.

Saijo, Y., Tintor, N., Lu, X., Rauf, P., Pajerowska-Mukhtar, K., Häweker, H., Dong, X., Robatzek, S., and Schulze-Lefert, P. 2009. Receptor quality control in the endoplasmic reticulum for plant innate immunity. EMBO J. 28:3439-3449.

Schmidt, R., Mieulet, D., Hubberten, H. M., Obata, T., Hoefgen, R., Fernie, A. R., Fisahn, J., San Segundo, B., Guiderdoni, E., Schippers, J. H., and Mueller-Roeber, B. 2013. Salt-responsive ERF1 regulates reactive oxygen species-dependent signaling during the initial response to salt stress in rice. Plant Cell 25:2115-2131.

Schulze, B., Mentzel, T., Jehle, A. K., Mueller, K., Beeler, S., Boller, T. Felix, G., and Chinchilla, D. 2010. Rapid heteromerization and phosphorylation of ligand-activated plant transmembrane receptors and their associated kinase BAK1. J. Biol. Chem. 285:9444-9451.

Segonzac, C., Feike, D., Gimenez-Ibanez, S., Hann, D. R., Zipfel, C., and Rathjen, J. P. 2011. Hierarchy and roles of pathogen-associated molecular pattern-induced responses in Nicotiana benthamiana. Plant Physiol. 156:687-699.

Serrano, M., Kanehara, K., Torres, M., Yamada, K., Tintor, N., Kombrink, E., Schulze-Lefert, P., and Saijo, Y. 2012. Repression of sucrose/ ultraviolet B light-induced flavonoid accumulation in microbeassociated molecular pattern-triggered immunity in Arabidopsis. Plant Physiol. 158:408-422.

Serrano, M., Robatzek, S., Torres, M., Kombrink, E., Somssich, I. E., Robinson, M., and Schulze-Lefert, P. 2007. Chemical interference of pathogen-associated molecular pattern-triggered immune responses in Arabidopsis reveals a potential role for fatty-acid synthase type II complex-derived lipid signals. J. Biol. Chem. 282:6803-6811.
Sharma, N., Bender, Y., Boyle, K., and Fobert, P. R. 2013. High-level expression of sugar inducible gene2 (HSI2) is a negative regulator of drought stress tolerance in Arabidopsis. BMC Plant Biol. 13:170.

Sørensen, B. B., Ehrnsberger, H. F., Esposito, S., Pfab, A., Bruckmann, A., Hauptmann, J., Meister, G., Merkl, R., Schubert, T., Längst, G., Melzer, M., Grasser, M., and Grasser, K. D. 2017. The Arabidopsis THO/TREX component TEX1 functionally interacts with MOS11 and modulates mRNA export and alternative splicing events. Plant Mol. Biol. 93:283-298.

Stegmann, M., Monaghan, J., Smakowska-Luzan, E., Rovenich, H., Lehner, A., Holton, N., Belkhadir, Y., and Zipfel, C. 2017. The receptor kinase FER is a RALF-regulated scaffold controlling plant immune signaling. Science 355:287-289.

Sun, Y., Li, L., Macho, A. P., Han, Z., Hu, Z., Zipfel, C., Zhou, J. M., and Chai, J. 2013. Structural basis for flg22-induced activation of the Arabidopsis FLS2-BAK1 immune complex. Science 342:624-628.

Takagi, H., Tamiru, M., Abe, A., Yoshida, K., Uemura, A., Yaegashi, H., Obara, T., Oikawa, K., Utsushi, H., Kanzaki, E., Mitsuoka, C., Natsume, S., Kosugi, S., Kanzaki, H., Matsumura, H., Urasaki, N., Kamoun, S., and Terauchi, R. 2015. MutMap accelerates breeding of a salt-tolerant rice cultivar. Nat. Biotechnol. 33:445-449.

Tintor, N., Ross, A., Kanehara, K., Yamada, K., Fan, L., Kemmerling, B., Nürnberger, T., Tsuda, K., and Saijo, Y. 2013. Layered pattern receptor signaling via ethylene and endogenous elicitor peptides during Arabidopsis immunity to bacterial infection. Proc. Natl. Acad. Sci. U.S.A. 110:6211-6216.

Tsukagoshi, H., Saijo, T., Shibata, D., Morikami, A., and Nakamura, K. 2005. Analysis of a sugar response mutant of Arabidopsis identified a novel B3 domain protein that functions as an active transcriptional repressor. Plant Physiol. 138:675-685.

Wan, J., Zhang, X. C., Neece, D., Ramonell, K. M., Clough, S., Kim, S. Y., Stacey, M. G., and Stacey, G. 2008. A LysM receptor-like kinase plays a critical role in chitin signaling and fungal resistance in Arabidopsis. Plant Cell 20:471-481.

Weigel, D., and Glazebrook, J. 2002. Arabidopsis: A Laboratory Manual. Cold Spring Harbor Laboratory Press, Cold Spring Harbor, NY, U.S.A.

Wierzba, M. P., and Tax, F. E. 2016. An allelic series of bakl mutations differentially alter birl cell death, immune response, growth, and root development phenotypes in Arabidopsis thaliana. Genetics 202:689-702.

Xu, J., Xie, J., Yan, C., Zou, X., Ren, D., and Zhang, S. 2014. A chemical genetic approach demonstrates that MPK3/MPK6 activation and NADPH oxidase-mediated oxidative burst are two independent signaling events in plant immunity. Plant J. 77:222-234.

Yamada, K., Saijo, Y., Nakagami, H., and Takano, Y. 2016. Regulation of sugar transporter activity for antibacterial defense in Arabidopsis. Science 354:1427-1430.

Yelina, N. E., Smith, L. M., Jones, A. M., Patel, K., Kelly, K. A., and Baulcombe, D. C. 2010. Putative Arabidopsis THO/TREX mRNA export complex is involved in transgene and endogenous siRNA biosynthesis. Proc. Natl. Acad. Sci. U.S.A. 107:13948-13953.

Zhang, H., Hong, Y., Huang, L., Li, D., and Song, F. 2016. Arabidopsis AtERF014 acts as a dual regulator that differentially modulates immunity against Pseudomonas syringae pv. tomato and Botrytis cinerea. Sci. Rep. 6:30251.

Zhang, H., Huang, L., Dai, Y., Liu, S., Hong, Y., Tian, L., Huang, L., Cao, Z., Li, D., and Song, F. 2015. Arabidopsis AtERF15 positively regulates immunity against Pseudomonas syringae pv. tomato DC3000 and Botrytis cinerea. Front. Plant Sci. 6:686.

Zhang, J., Li, W., Xiang, T., Liu, Z., Laluk, K., Ding, X., Zou, Y., Gao, M., Zhang, X., Chen, S., Mengiste, T., Zhang, Y., and Zhou, J. M. 2010. Receptor-like cytoplasmic kinases integrate signaling from multiple plant immune receptors and are targeted by a Pseudomonas syringae effector. Cell Host Microbe 7:290-301.

Zhang, Z., Tateda, C., Jiang, S. C., Shrestha, J., Jelenska, J., Speed, D. J., and Greenberg, J. T. 2017. A suite of receptor-like kinases and a putative mechano-sensitive channel are involved in autoimmunity and plasma membrane-based defenses in Arabidopsis. Mol. Plant-Microbe Interact. 30:150-160.

Zhu, Y., Schluttenhoffer, C. M., Wang, P., Fu, F., Thimmapuram, J., Zhu, J. K., Lee, S. Y., Yun, D. J., and Mengiste, T. 2014. CYCLINDEPENDENT KINASE8 differentially regulates plant immunity to fungal pathogens through kinase-dependent and -independent functions in Arabidopsis. Plant Cell 26:4149-4170.

Zipfel, C., Kunze, G., Chinchilla, D., Caniard, A., Jones, J. D., Boller, T., and Felix, G. 2006. Perception of the bacterial PAMP EF-Tu by the receptor EFR restricts Agrobacterium-mediated transformation. Cell 125:749-760.

Zipfel, C., Robatzek, S., Navarro, L., Oakeley, E. J., Jones, J. D., Felix, G., and Boller, T. 2004. Bacterial disease resistance in Arabidopsis through flagellin perception. Nature 428:764-767. 